\title{
Multiple Fano resonances Based on the Rectangular cavity resonator and crescent-shaped cavity resonator
}

Jun Zhu ( $\square$ zhujun1985@gxnu.edu.cn )

Guangxi Normal University

Ge Wang

Guangxi Normal University

\section{Research}

Keywords: Water Content in Heavy Oil, Crescent-shaped Cavity Resonator, Fano resonances

Posted Date: October 6th, 2020

DOI: https://doi.org/10.21203/rs.3.rs-84286/v1

License: (c) (i) This work is licensed under a Creative Commons Attribution 4.0 International License.

Read Full License 


\section{Multiple Fano resonances Based on the Rectangular cavity resonator and crescent-shaped cavity resonator \\ JUN ZHU ${ }^{1 *}$, GE WANG ${ }^{1}$, \\ 1 College of Electronic Engineering, Guangxi Normal University, Guilin 541004,China}

*Correspondence and requests for materials should be addressed to JUN ZHU (zhujun1985@gxnu.edu.cn)

Abstract: World is rich in unconventional oil and various alternatives to petroleum. However, conventional oil production declines so quickly that it is likely these unconventional oil resources cannot be put into production fast enough, and thus will not be compensated sufficiently. We realize detecting rapid detection of water content in heavy oil. The waveguide consists of a metal-insulator-metal (MIM) waveguide, rectangular cavity resonator, and crescent-shaped cavity resonator. The effects of the coupling distance, geometry of the crescent-shaped cavity resonator and its rotation angle, and length and width of the rectangular cavity resonator on the Fano resonance lines were numerically analyzed. Multiple Fano resonances can be produced as the rotation angle of the crescent-shaped cavity resonator is adjusted, and the sensor's refractive index sensitivity was found to be $935.71 \mathrm{~nm} / \mathrm{RIU}$. By measuring the water content in heavy oil, we found that the Fano resonance lines shift toward shorter wavelengths as the volume fraction of water content increases. The detection resolution in heavy oil $1.79 \times 10^{-9}$. The results presented here show that water content in heavy oil can be calculated using the measured change in the Fano resonance wavelength.

Keywords: Water Content in Heavy Oil; Crescent-shaped Cavity Resonator; Fano resonances

\section{INTRODUCTION}

World is rich in unconventional oil and various alternatives to petroleum. However, conventional oil production declines so quickly that it is likely that these unconventional oil resources cannot be put into production fast enough, and thus will not be compensated sufficiently [1-5]. Surface plasmon polaritons (SPPs) are electromagnetic fields that propagate along a metal-insulator interface. Because they can modulate light at the nanometer scale, they can generate extremely strong local electromagnetic fields and break the diffraction limit. They have attracted a great deal of research interest [6-9], and they could be used in unique devices. So far, various plasma devices have been proposed for measuring water content, such as optical filters [10], wavelength demultiplexer [11], and information modulators [12], all of which could be integrated into system-on-a-chip devices. In addition, the Fano resonance [13-15] has become the focus of SPP devices due to its considerable asymmetry in line-shape. In addition, the resonance wavelength and peak intensity are highly sensitive to small disturbances [16-18]. And because the MIM waveguide structure has a strong restraint ability on SPPs, and the Fano resonance effect generated in the MIM waveguide. It is extremely sensitive to changes in environmental refractive index, structural parameters, and polarization state of incident light. Combining the advantages of both, it has become a new research direction of SPP devices. In recent years, due to the characteristic of Fano resonance, a lot of research has been done on it. For example, based on the plasmon hybridization theory, He et al. [19] studied the Fano resonance in an asymmetric ring and its sensing characteristics by offsetting the centers of the inner and outer walls. They obtained sensitivity of $626 \mathrm{~nm} /$ RIU [19]. In 2018, Guo et al. [20] proposed a multi-channel refractive index sensor that takes advantage of coupling between a sub wavelength metal-insulator-metal (MIM) waveguide and a tangent-ring cavity resonator. This sensor exhibited sensitivity $880 \mathrm{~nm} / \mathrm{RIU}$.

To realize rapid detection of water content in heavy oil, we designed an MIM waveguide structure composed of a rectangular cavity resonator and a crescent-shaped cavity resonator. The finite element method was used to numerically simulate the structure under a perfectly matched layer (PML) boundary conditions [21,22]. Simulations were performed with various coupling distances, geometry of the rectangular and crescent-shaped resonators, and rotation angles, and the effects of these changes on the transmission characteristics were calculated. We obtained a relationship between the water content in heavy oil and the Fano resonance wavelength in the sensor. The structure presented here could also be used for the detection of other trace elements in liquids.

\section{MODEL AND CALCULATION METHOD}

\subsection{MODEL}

The structure diagram is as follows, as shown in Figure 1. The width and height of the rectangular cavity resonator are $W$ and $H$, respectively. $D=50 \mathrm{~nm}$ is the width of the linear waveguide and $G$ is the coupling distance between the rectangular and crescent-shaped resonators. The transmittance $T$ at the output port is the ratio of the output power $P_{\text {out }}$ to the input power $P_{\text {in }}$, i.e., $T=P_{\text {out }} / P_{\text {in }}$, where $P_{\text {in }}=\int$ Poavxd $_{1}, P_{\text {out }}=\int$ Poavxd $_{2}$, and Poavx is the time-averaged power flow in the axial direction. 

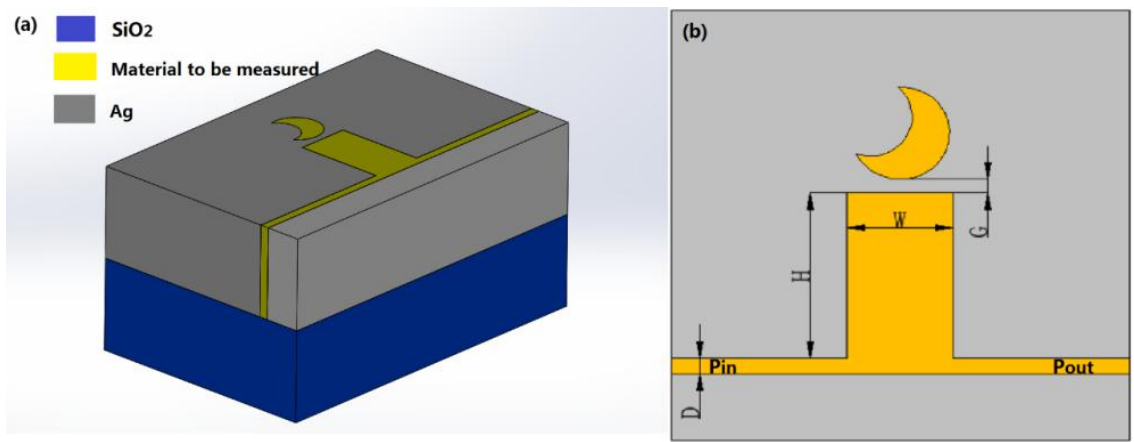

Fig. $13 \mathrm{D}$ and 2D renderings of the system

\subsection{Fano resonance}

As shown in Figure 2, R1 and R2 are the radii of the inner and outer arcs of the crescent-shaped cavity, respectively; $d$ is the distance between the two centers of the arcs, and $\alpha$ is the rotation angle. Figures 3a-m shows the transmission spectra of $\alpha$ in $30^{\circ}$ increments ranging from $0^{\circ}$ to $360^{\circ}$. Figure 3a shows a transmission spectrum with asymmetric Fano line width in the coupled rectangular and crescent resonators. The peak in the spectrum is Peak I, at this time $\alpha=0^{0}$. Comparing Figures 3a and 3b, one can see a new Fano resonance line shape appears on the right side of the original transmission spectrum as $\alpha$ increases from $0^{\circ}$ to $30^{\circ}$. The valley on the right is referred to as Dip I. Figure 3b-e shows that the position and transmittance of Peak I remain constant while Dip I is redshifted as $\alpha$ increases from $30^{\circ}$ to $120^{\circ}$. Figure 3i-l shows the peak position and transmittance of Peak I remain constant, while Dip I is blueshifted, as $\alpha$ increases from $240^{\circ}$ to $330^{\circ}$. Furthermore, Figures $3 \mathrm{a}, 3 \mathrm{~g}$, and $3 \mathrm{~m}$ show that the transmission spectra for $\alpha=0^{\circ}, 180^{\circ}$, and $360^{\circ}$ are the same. We can also see that the transmission spectra in Figures $3 \mathrm{~b}\left(\alpha=30^{\circ}\right)$ and $31\left(\alpha=330^{\circ}\right), 3 \mathrm{c}\left(\alpha=60^{\circ}\right)$ and $3 \mathrm{k}\left(\alpha=300^{\circ}\right), 3 \mathrm{~d}\left(\alpha=90^{\circ}\right)$ and $3 \mathrm{j}\left(\alpha=270^{\circ}\right), 3 \mathrm{e}\left(\alpha=120^{\circ}\right)$ and $3 \mathrm{i}\left(\alpha=240^{\circ}\right)$, and $3 \mathrm{f}\left(\alpha=150^{\circ}\right)$ and $3 \mathrm{~h}\left(\alpha=210^{\circ}\right)$ are the same. Based on the above results of the transmissions and FWHM (full width at half maximum), we selected $\alpha=60^{\circ}$. Finally, the magnetic field diagram of $\mathrm{A}, \mathrm{B}$ and $\mathrm{C}$ at the three resonance wavelengths in Fig. $3 \mathrm{c}$ is given, as shown in the lower right corner of Fig. 3.

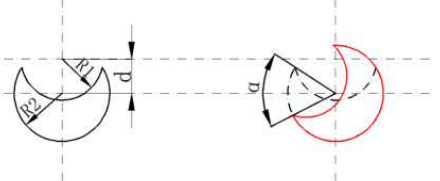

Fig. 2 Schematic diagram showing the structure of the crescent-shaped cavity resonator.
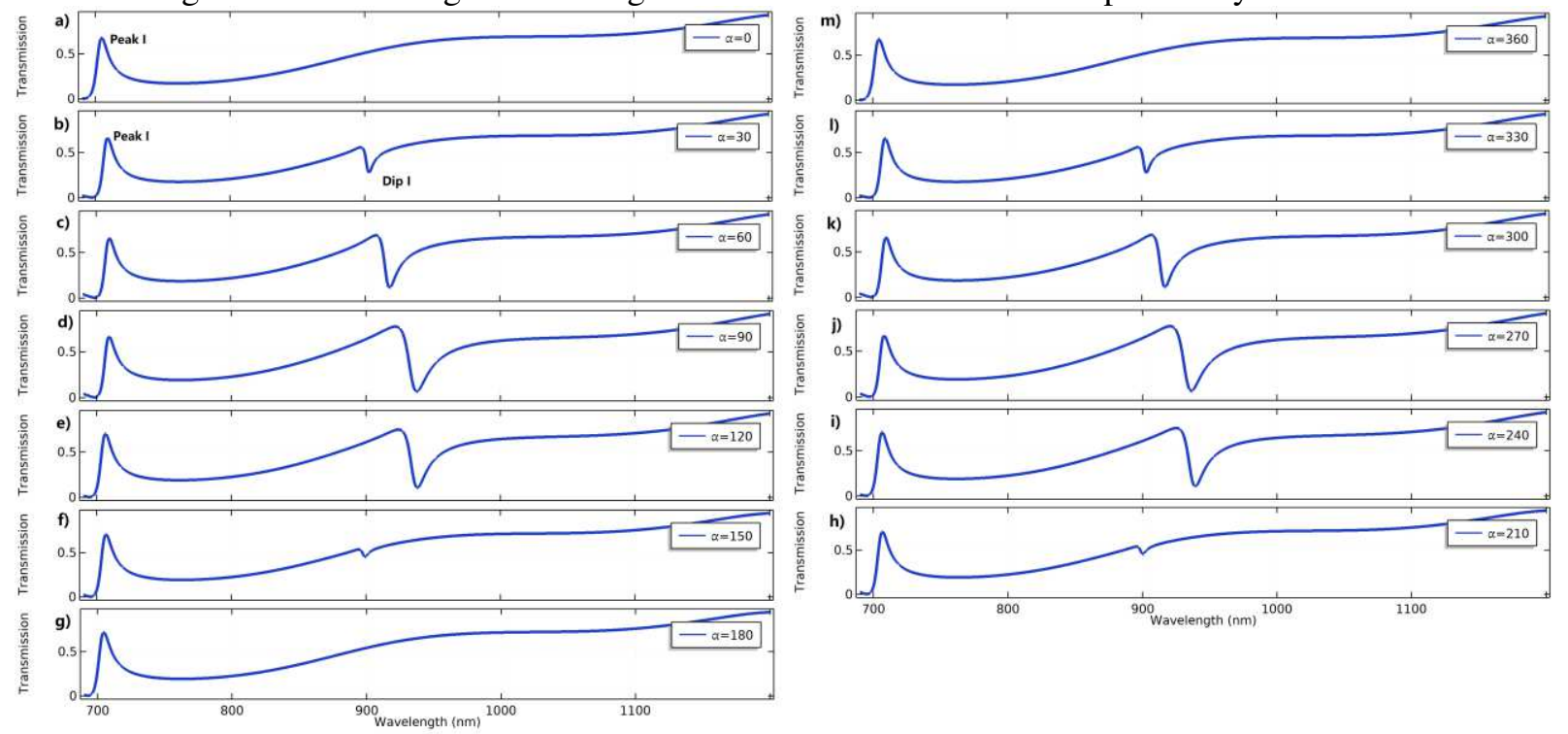

Fig. 3 Transmission spectra from the designed structure at different the rotation angle $\alpha$. Figure A-C is the magnetic field distribution diagram 


\section{Parameters on Fano resonanc}

\subsection{Effect of parameters on Fano resonance line}

In this section we discuss how changes in the structure's geometry affect the Fano resonance lines. Figure 4 shows the transmission spectra and FWHM of the Fano resonance lines as $R 2$ (radius of the outer arc of the crescent-shaped resonator) increases from 120 to $160 \mathrm{~nm}$. The resolution of the sensor refers to the smallest change in the refractive index of the sample that can be detected by the sensing system and is expressed in terms of FWHM. The other parameters were set $\mathrm{R} 1=120 \mathrm{~nm}, \mathrm{~d}=100 \mathrm{~nm}, \mathrm{~W}=300 \mathrm{~nm}, \mathrm{H}=500 \mathrm{~nm}, \mathrm{G}=10 \mathrm{~nm}, \alpha=60^{\circ}$, and $\mathrm{n}=1$. Figure $4 \mathrm{a}$ shows that, as $R 2$ increases, the position of Peak I remains unchanged, while the transmittance peak of Peak I decrease slightly from 0.678 to 0.67 . Meanwhile, Dip I is redshifted from 803 to $1046 \mathrm{~nm}$, and the transmittance of Dip I increased from 0.036 to 0.155. Figure 4b shows the change in FWHM of the Fano resonance line as $R 2$ increases from 120 to $160 \mathrm{~nm}$. As $R 2$ increases from 120 to $130 \mathrm{~nm}$, the FWHM of Peak I first decreases from 10 to $9 \mathrm{~nm}$ and then remains constant. As $R 2$ increases from 120 to $140 \mathrm{~nm}$, the FWHM of Dip I decreases rapidly from 30 to $13 \mathrm{~nm}$, and the decline is smaller as $R 2$ increases from 140 to $160 \mathrm{~nm}$. Based on these transmittance and FWHM results, we selected $R 2=150 \mathrm{~nm}$.
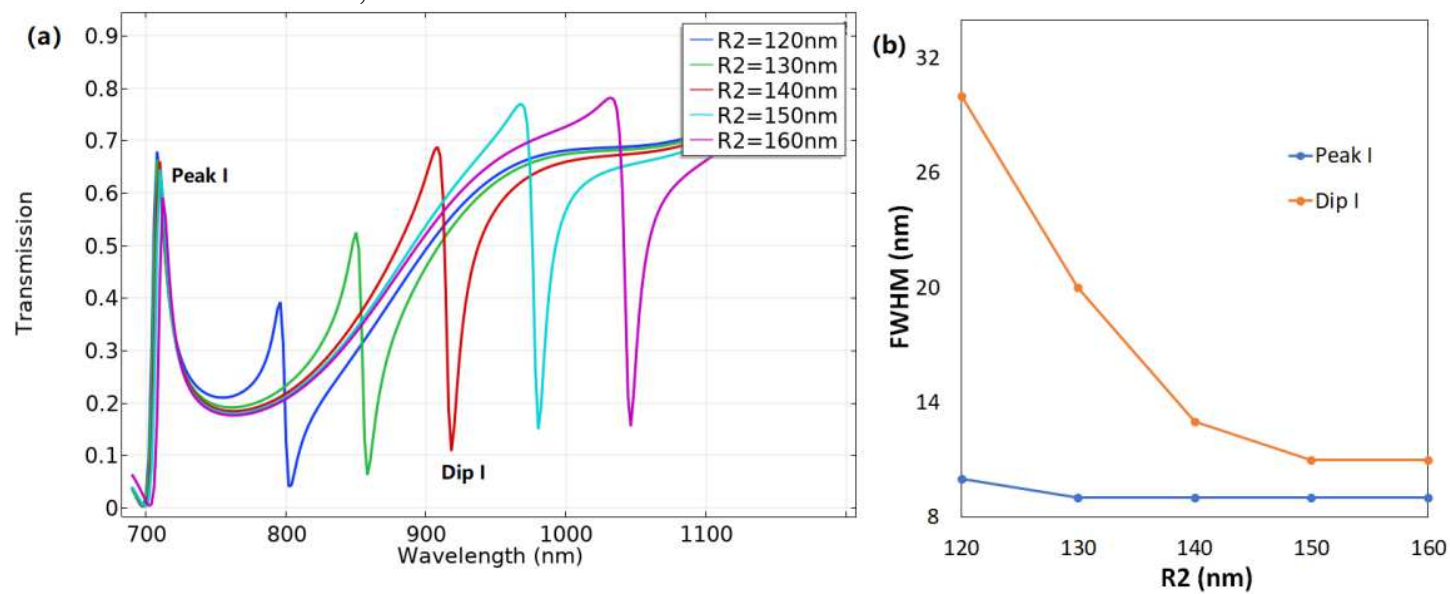

Fig.4 (a) The transmission spectra of R2 ; $\quad$ (b) FWHM with the Fano resonance lines.

Figure $5 \mathrm{a}$ shows the transmission spectra as $R 1$ (radius of the inner arc of the crescent-shaped resonator) increases from 100 to $160 \mathrm{~nm}$. The other parameters were set $R 2=150 \mathrm{~nm}, d=100 \mathrm{~nm}, W=300 \mathrm{~nm}, H=$ $500 \mathrm{~nm}, G=10 \mathrm{~nm}, \alpha=60^{\circ}$, and $n=1$. As $R 1$ increases, the peak wavelength and transmittance of Peak I remain unchanged, while Dip I is slightly redshifted, and its transmission decreases from 0.17 to 0.1 . Furthermore, as $R 1$ increases, the FWHM of Dip I gradually increases. When $R 1$ is between 130 and $140 \mathrm{~nm}$, the FWHM of Dip I remains at $12 \mathrm{~nm}$. The FWHM of Peak I remains at $9 \mathrm{~nm}$, as shown in Figure 5b. Based on these transmittance and FWHM results, we selected $R 1=140 \mathrm{~nm}$. Figure $5 \mathrm{c}$-d shows the transmission spectra and FWHM of the Fano resonance lines as $d$ (distance between the centers of the inner and outer arcs of the crescent-shaped resonator) increases from 80 to $120 \mathrm{~nm}$, with the other parameters set to $R 2=150 \mathrm{~nm}, R 1=140 \mathrm{~nm}, W=300 \mathrm{~nm}, H=$ $500 \mathrm{~nm}, G=10 \mathrm{~nm}, \alpha=60^{\circ}$, and $n=1$. Figure 5c shows that the wavelength and transmittance of Peak I remain virtually constant as $d$ increases, while the peak wavelength of Dip I is blueshifted from 1081 to $927 \mathrm{~nm}$, and its transmission varies around 0.1. Figure 5d shows that the FWHM of Peak I remains at $9 \mathrm{~nm}$, while the FWHM of Dip I decreases first and then increases as $d$ increases. Based on these transmittance and FWHM results, we selected $d=110 \mathrm{~nm}$. From these simulation results, we can see that changing the radii of the inner and outer arcs in the crescent-shaped resonator, as well as the distance between the centers of the inner and outer arcs, induce significant changes on Dip I but only small changes in Peak I. 

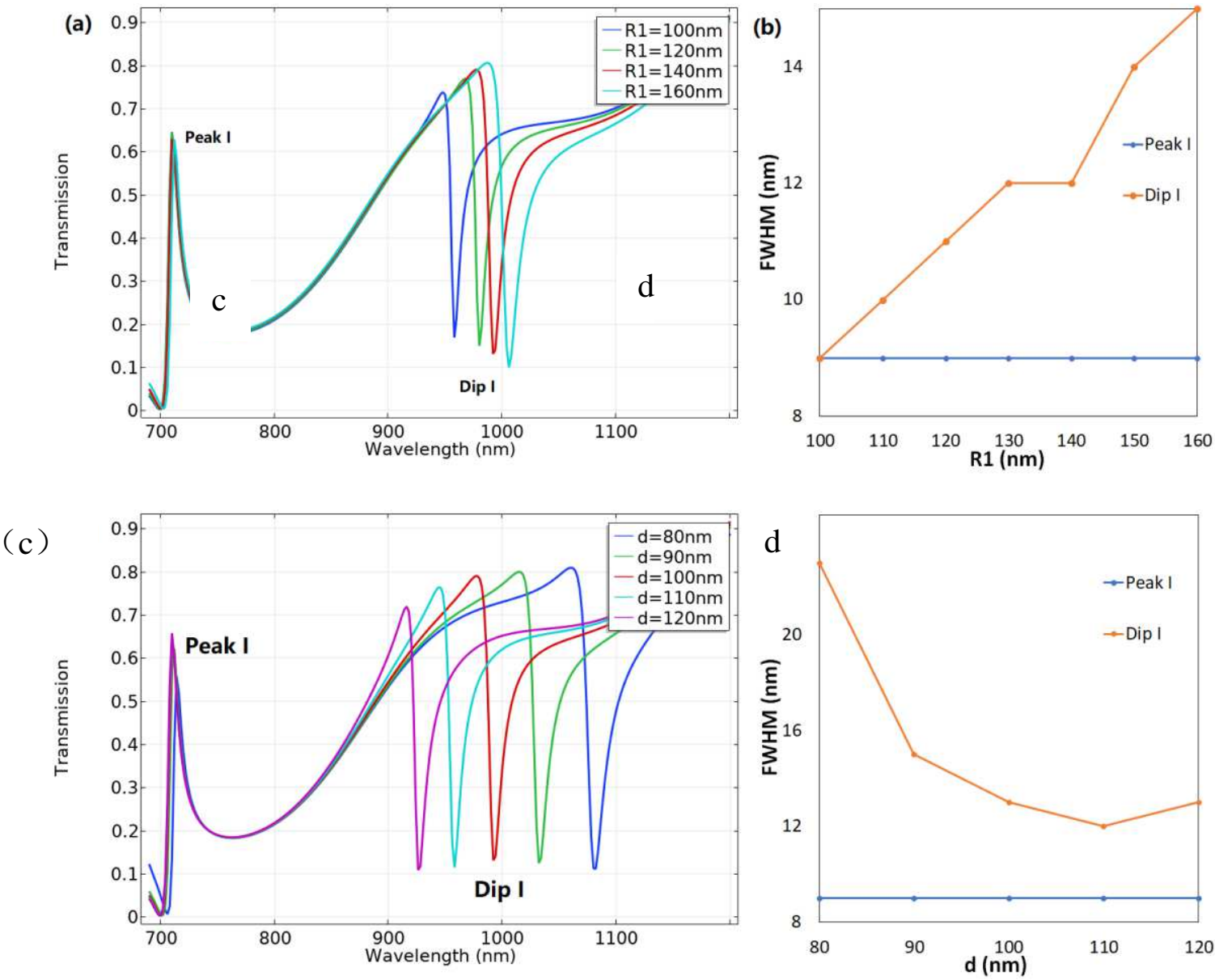

Fig. 5 (a) The transmission spectra of R1 (b) R1 and FWHM. (c) Transmission spectra of $d$. (d) $d$ and FWHM

Figure 6a shows how the transmission spectra change as $W$ increases from 280 to $320 \mathrm{~nm}$. The other parameters were set to $R 2=150 \mathrm{~nm}, R 1=140 \mathrm{~nm}, d=110 \mathrm{~nm}, H=500 \mathrm{~nm}, G=10 \mathrm{~nm}, \alpha=60^{\circ}$, and $n=1$. As can be seen from the figure, the resonance wavelength of Peak I is redshifted from 675 to $746 \mathrm{~nm}$, and the transmittance of Peak I increases from 0.51 to 0.7 as $W$ increases. Meanwhile, the position of Dip I remains unchanged and the transmittance of Dip I varies around 0.1. Figure 6b shows the FWHM of the Fano resonance lines as $W$ increases from 280 to $360 \mathrm{~nm}$. The FWHM of Peak I first increases, then levels off, and subsequently increases again. The FWHM of Dip I consistently decreases. We can clearly see that, when $W=330 \mathrm{~nm}$, the FWHM of Peak I and the FWHM of Dip I are both $10 \mathrm{~nm}$. Based on these transmittance and FWHM results, we selected $W=330 \mathrm{~nm}$. Figure 7a shows the transmission spectra as $H$ increasing from 460 to $540 \mathrm{~nm}$, with the other parameters set to $R 2=$ $150 \mathrm{~nm}, R 1=140 \mathrm{~nm}, d=110 \mathrm{~nm}, W=330 \mathrm{~nm}, G=10 \mathrm{~nm}, \alpha=60^{\circ}$, and $n=1$. One can see from the figure that the resonance wavelength position of Peak I is slightly blueshifted from 764 to $762 \mathrm{~nm}$ and its transmission decreases from 0.797 to 0.677 as $H$ increases. The resonance wavelength of Dip I is slightly redshifted from 956 to $959 \mathrm{~nm}$, and its transmission increases from 0.129 to 0.146. Figure 7b shows that the FWHM of Peak I and Dip I change in the same manner as $H$ increases: they first decrease and then level off. Based on the results of transmission and FWHM, we selected $H=490 \mathrm{~nm}$. In the above, we analyze the effect of the width and height of the rectangular cavity on the Fano resonance line. One can see that chang $W$ significantly changes Peak I and creates small changes in Dip I. One can also see that changing $H$ of the rectangular cavity resonator changes both Peak I and Dip I. 

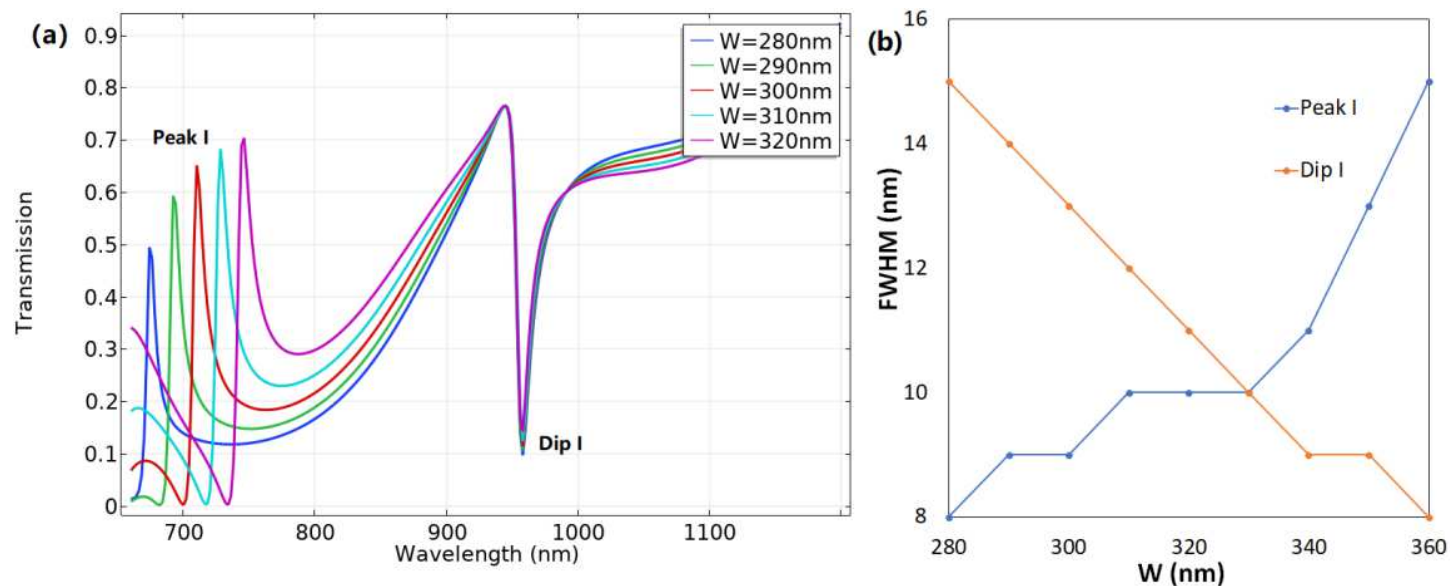

Fig. 6 (a) Transmission spectra a of the rectangular cavity resonator (b) $W$ and FWHM
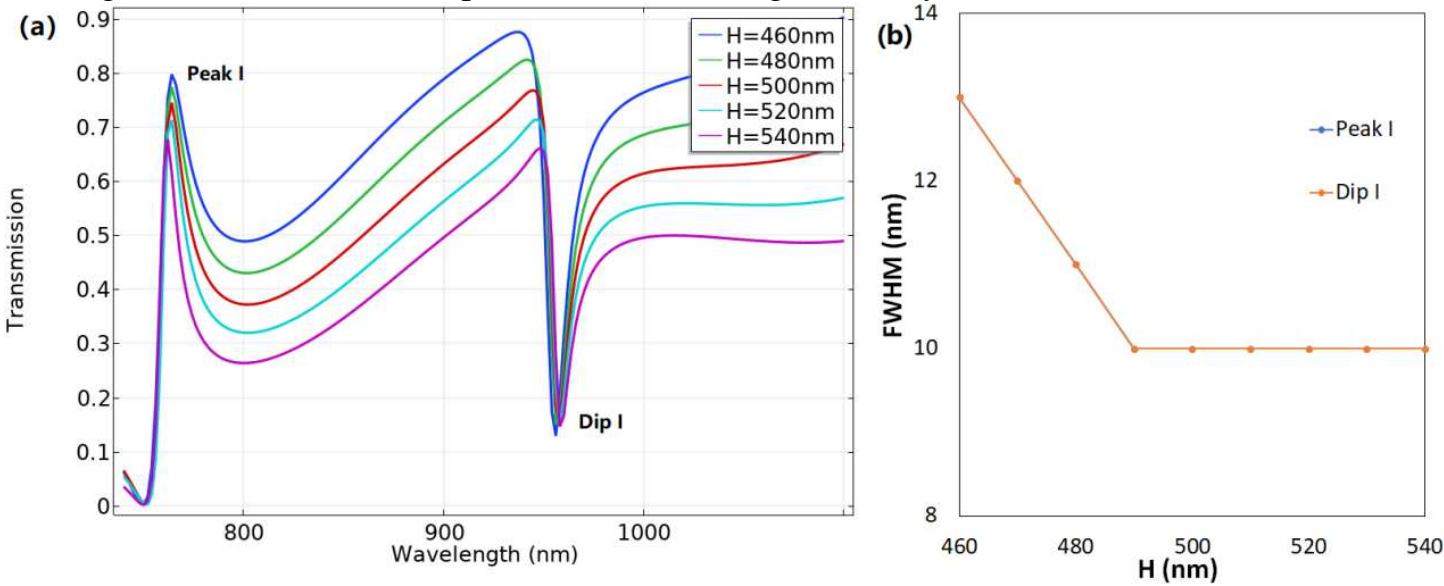

Fig. 7 (a) Transmission spectra of the height $H$ (b) $H$ and FWHM

Finally, Figure 8 shows the transmission spectra and FWHM of the Fano resonance lines as $G$ (coupling distance between the rectangular resonator and the crescent-shaped resonator) increases from 0 to $20 \mathrm{~N}$., The other parameters were set to $R 2=150 \mathrm{~nm}, R 1=140 \mathrm{~nm}, d=110 \mathrm{~nm}, W=330 \mathrm{~nm}, H=490 \mathrm{~nm}, \alpha=60^{\circ}$, and $n=1$. Figure $8 \mathrm{a}$ shows that the position of the Fano resonance line is blueshifted as $G$ increases. As $G$ increases from 0 to $5 \mathrm{~nm}$, the resonance wavelength of Peak I shift from 782 to $767 \mathrm{~nm}$. As $G$ increases from 5 to $20 \mathrm{~nm}$, the resonance wavelength of Peak I shift from 767 to $759 \mathrm{~nm}$. As $G$ increases from 0 to $5 \mathrm{~nm}$, the resonance wavelength of Dip I shifts from 1070 to $982 \mathrm{~nm}$, while the resonance wavelength of Dip I shifts from 982 to $941 \mathrm{~nm} G$ increases from 5 to $20 \mathrm{~nm}$. At $G=0 \mathrm{~nm}$, the FWHM of Dip I is $180 \mathrm{~nm}$, which is a very large value, while the FWHM of Peak I is 9 $\mathrm{nm}$. Figure $8 \mathrm{~b}$ shows the change of the FWHM of the Fano resonance lines as $G$ increases from 5 to $20 \mathrm{~nm}$. We see that the FWHM of Peak I change slowly, while the FWHM of Dip I decreases rapidly. Based on the results of transmission and FWHM, we selected $G=10 \mathrm{~nm}$.
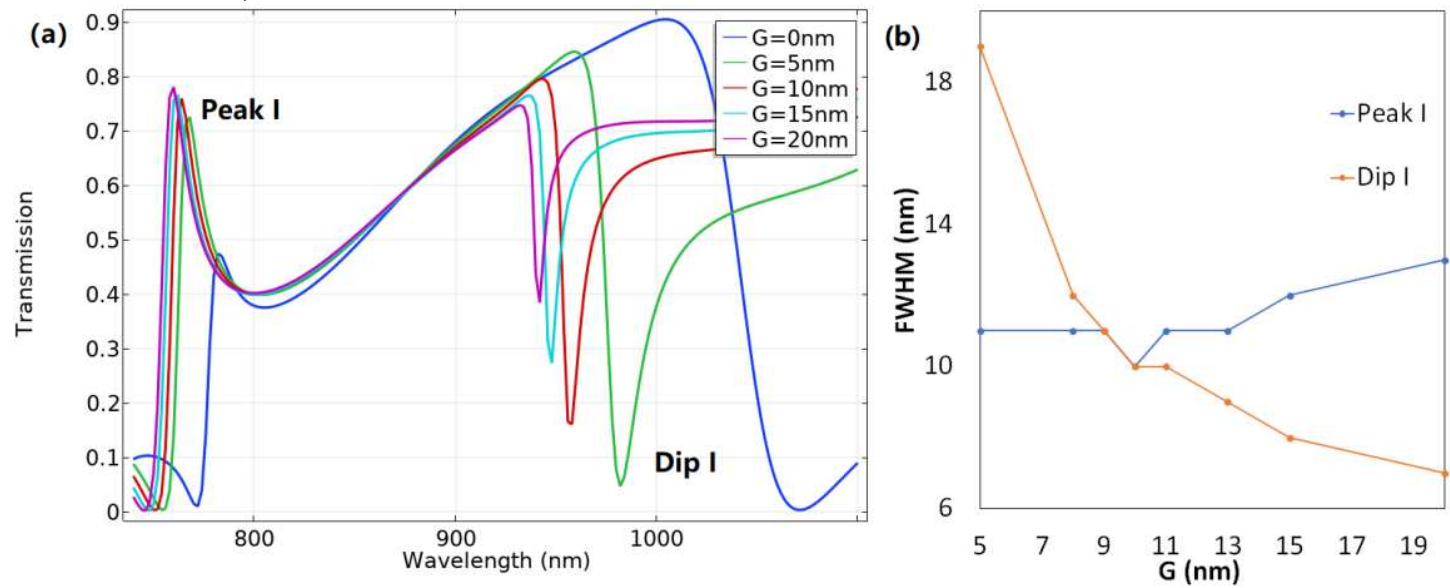

Fig. 8 (a) Transmission spectra with distance $G$ (b) $G$ and FWHM 


\subsection{Analysis of sensitivity of Fano resonance}

Figure 9a shows the transmission spectra as the refractive index of the material under test, which fills the structure, increases from 1 to 1.06 . We used a linear fit to the Fano resonance wavelength as function of $n$, yielding $S=$ $750 \mathrm{~nm} / R I U$ for Peak I and $S=935.71 \mathrm{~nm} / R I U$ for Dip I. The FWHM of Peak I and Dip I are $10 \mathrm{~nm}$. The FOM values for Peak I and Dip I are 75 and 93.571, respectively. Finally, we offer a comparison with other research results in Table I. The structure proposed in this paper has significantly greater refractive index sensitivity.
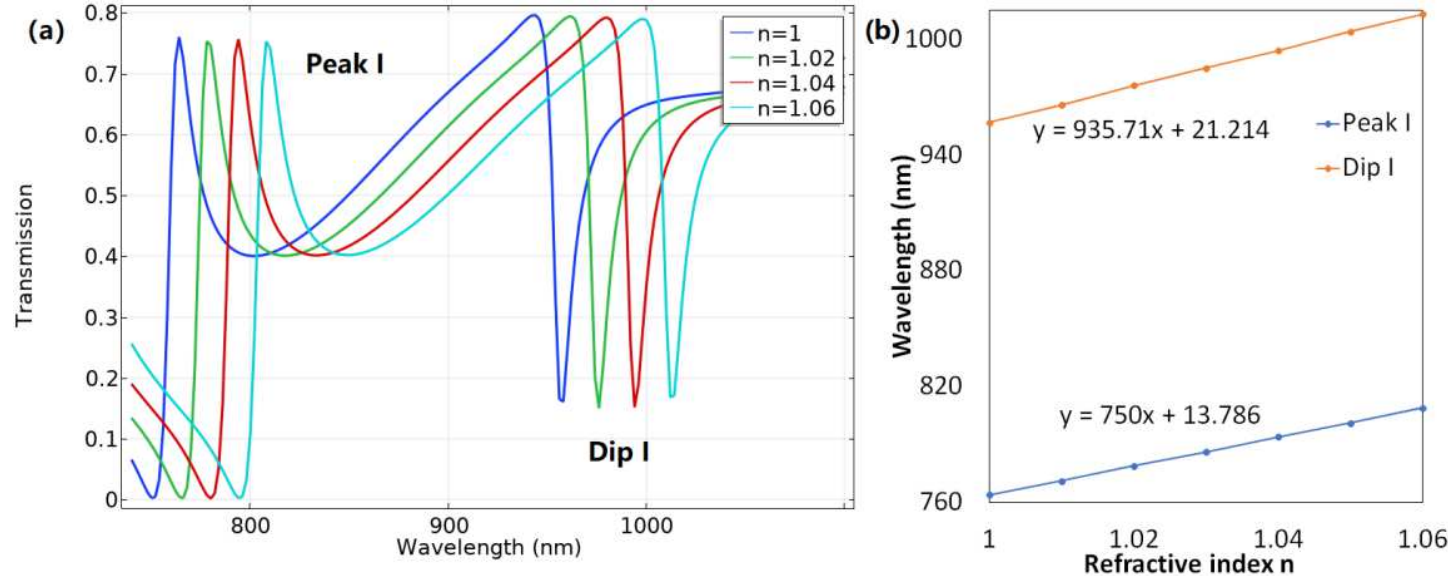

Fig. 9 (a) Transmission spectra as the refractive index $n$. (b) Fano resonance wavelength with refractive index

\section{APPLICATION TO TESTING ON WATER CONTENT IN HEAVY OIL}

World is rich in unconventional oil and various alternatives to petroleum. Water in heavy oil has a great impact on combustion and metallurgical reaction processes in smelting furnaces, and the water content will also have a certain effect on normal equipment operation. Figure 10 shows the transmission spectra for heavy oils with water content of $0 \%, 20 \%, 40 \%$, and $60 \%$. The results were obtained with the designed structure filled with heavy oil. One can see that the Fano resonance line moves to shorter wavelengths with little changes in transmission as the water content increases. We have compiled data for the volume percentage of water content with the corresponding refractive index and Fano resonance wavelength into Table II.

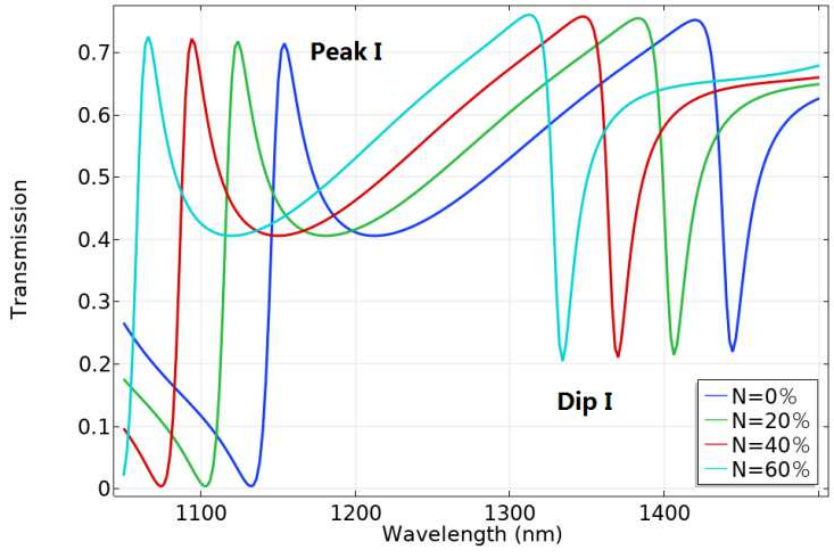

Fig. 10 Heavy oils with different water content

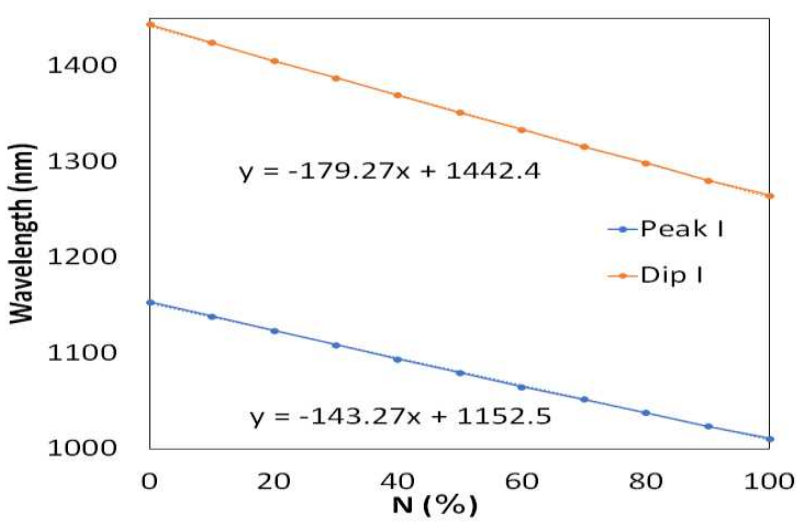

Fig. 11. Volume fraction of water with the Fano resonance wavelength.

The data in Table II were to fit to a linear function of water volume fraction, as shown in Figure 11. The fitting functions for Peak I and Dip I are $\lambda_{\text {Peak I }}=-143.27 \mathrm{~N}+1152.5$ and $\lambda_{\text {Dip I }}=-179.27 \mathrm{~N}+1442.4$, respectively, with coefficients of determination as high as 0.999 . The water volume fraction resolution in heavy oil is $1.79 \times 10^{-9}$.

Table II. Water in heavy oil corresponds to the refractive index and resonance wavelength.

\begin{tabular}{llll}
\hline $\mathrm{N}$ & $n_{c}$ & $\lambda_{\text {Peak I }}(\mathrm{nm})$ & $\lambda_{\text {Dip I }}(\mathrm{nm})$ \\
\hline $0 \%$ & 1.520 & 1154 & 1444 \\
$10 \%$ & 1.500 & 1139 & 1425 \\
$20 \%$ & 1.480 & 1124 & 1406 \\
$30 \%$ & 1.460 & 1109 & 1388 \\
$40 \%$ & 1.441 & 1094 & 1370 \\
$50 \%$ & 1.421 & 1080 & 1352
\end{tabular}




$\begin{array}{llll}60 \% & 1.403 & 1065 & 1334 \\ 70 \% & 1.384 & 1052 & 1316 \\ 80 \% & 1.366 & 1038 & 1299 \\ 90 \% & 1.348 & 1024 & 1281 \\ 100 \% & 1.330 & 1011 & 1265\end{array}$

\section{Results and Discussion}

World is rich in unconventional oil and various alternatives to petroleum. Water in heavy oil has a great impact on combustion and metallurgical reaction processes in smelting furnaces, and the water content will also have a certain effect on normal equipment operation.The relationship between the structure's geometry and its transmission spectrum was studied in detail using FEM simulations. Multiple Fano resonances can be produced by changing the rotation angle of the crescent-shaped resonator. The refractive index sensitivities of the systems reported in [25], [19], and [20] are 596, 626, and $880 \mathrm{~nm} / \mathrm{RIU}$, respectively. With respect to these values, the refractive index sensitivities proposed in this paper are $56.9 \%, 49.5 \%$, and $6.3 \%$ greater, respectively.

Table I Sensitivity of similar devices reported in the literature.

\begin{tabular}{lc}
\hline References & Sensitivity (nm/RIU) \\
\hline [25] Zhidong, Zhang, Liang, & 596 \\
Luo, Chenyang, Xue, Wendong, \\
Shubin, Yan. Fano Resonance Based \\
on Metal-Insulator-Metal \\
Waveguide-Coupled Double \\
Rectangular Cavities for Plasmonic \\
Nanosensors[J]. Sensors, 2016, \\
16(5):642-652. \\
[19] He, J., Fan, C., Wang, J., \\
Ding, P., Cai, G., \& Cheng, Y. A \\
giant localized field enhancement \\
and high sensitivity in an asymmetric \\
ring by exhibiting Fano resonance \\
[J]. Journal of Optics, 2013, \\
15(2):025007. \\
Shu-Bin, Y., Liang, L., \\
[26] 626 \\
Chen-Yang, X. , \& Zhi-Dong, Z. A \\
Refractive Index Sensor Based on a \\
Metal-Insulator-Metal \\
Waveguide-Coupled Ring \\
Resonator[J]. Sensors, \\
15(11):29183-29191. \\
[20] Zicong, G., Kunhua, W., \\
Qinyang, H., Wenhui, L., Jiyan, L. , \\
\& Yihong, F. . Plasmonic \\
Multichannel Refractive Index \\
Sensor Based on Subwavelength \\
Tangent-Ring Metal-Insulator-Metal \\
Waveguide[J]. Sensors, 2018, \\
18(5):1348-1357. \\
This study \\
\hline
\end{tabular}

The main innovations are as follows:

1) A nano-scale refractive index sensor with a high sensitivity $935.71 \mathrm{~nm} / \mathrm{RIU}$.

2) The FOM as high as 935.71 can be constructed by filling the area reserved for material under test with materials that have different refractive index.

This structure was used to detect the water content in heavy oil. As the volume fraction of water increases, the Fano resonance line moves toward shorter wavelengths. The results show that the water content in heavy oil can be calculated using a measured change in the Fano resonance wavelength. The resolution of the sensor was found to be $1.79 \times 10^{-9}$. The structure designed in this paper provides a new way to measure liquid solvent contents in solutions. 


\section{Declarations}

Availability of data and materials: The experiments of our paper have no ethical issues. And the experimental protocols were approved by the Animal Care and Protection Committee of GuangxiNormal University.

Competing interests: No conflict of interest exits in the submission of this manuscript

Funding : This project is supported by National Natural Science Foundation of China (Grant No. 51965007),"One thousand Young and Middle-Aged College and University Backbone Teachers Cultivation Program" of Guangxi(2019), Guangxi Natural Science Foundation.

Authors' contributions: Ge Wang and JUN ZHU contributed equally to this work. Ge Wang and JUN ZHU drafted the manuscript. Cong hu participated in the design of the study and performed the statistical analysis.All authors read and approved the final manuscript.

Acknowledgements: Written informed consent was obtained from all the authors for publication of their individual details and accompanying images in this manuscript.

Authors' information : Jun ZHU and Wang, College of Electronic Engineering,Guangxi Normal University,Guilin 541004,China

\section{REFERENCES}

[1] Mikhail F. Limonov, Mikhail V. Rybin, Alexander N. Poddubny. Fano resonances in photonics[J]. Nature Photonics, 2017, 11(9):543-554.

[2] Tareq Al-Attas, Syed Ahmed Ali, Md Hasan Zahir. Recent Advances in Heavy Oil Upgrading using Dispersed Catalysts [J]. Energy \& Fuels, 2019.

[3] J H Sun, F S Zhang, Y W Wu. Overview of emulsified viscosity reducer for enhancing heavy oil recovery [J]. IOP Conference Series Materials Science and Engineering, 2019, 479:012009.

[4] Namsaraev, Z. B. , Kadiev, K. M. , Dandaev, A. U. , Barkhutova, D. D. , \& Ivanov, E. V. Combined Hydrothermal Conversion of Biomass (Algae and Aquatic Vegetation) from Lake Baikal Littoral Zone and Heavy-Oil Resids to Produce Biofuel [J]. 2018, 53(6):813-816.

[5] Wang S, Li A. Effect of water content and temperature on the rheological behavior of Caoqiao heavy oil [J]. Petroleum Science and Technology, 2018, 36(11):1-5.

[6] G.P. Kayukova, D.A. Feoktistov, A.N. Mikhailova. Influence of the Nature of Metals and Modifying Additives on Changes in the Structure of Heavy Oil in a Catalytic Aquathermolysis System[J]. Petroleum Chemistry, 2018, 58(3):190-196.

[7] Atian X, Jiuyun C, Yangyang C, Jihui L, Chunxiang L, Yongsheng Y, Jiangdong D. Capillarity-driven both light and heavy oil/water separation via combined system of opposite superwetting meshes [J]. Sep Purif Technol, 2019(215), 1-9.

[8] Xiaobei Zhang, Yong Yang, Haiyang Shao. Fano resonances in cone-shaped inwall capillary based microsphere resonator [J]. Optics Express, 2017, 25(2):615.

[9] Mariana del Pino, Virginia Fano, Paula Adamo. Growth velocity and biological variables during puberty in achondroplasia [J]. Journal of Pediatric Endocrinology \& Metabolism, 2018, 31(4):421-428.

[10] Mohammad Amin A, Elefano Elizabeth C, Leigh Darin. Use of Computer Simulation to Study Impact of Increasing Routine Test Volume on Turnaround Times of STAT Samples on ci8200 Integrated Chemistry and Immunoassay Analyzer[J]. Clinical Chemistry, 2020(10):10.

[11] Theofano Tikka, Constantina P. Yiannakis, Emma Stapleton. Spontaneous Vestibular Schwannoma Regression: A Case-Control Study[J]. Otology \& Neurotology, 2018, 39(10):1.

[12] Gabriela P. F. Arrifano, Mathieu P. Lichtenstein, José Rogério Souza-Monteiro. Clarified Açaí ( Euterpe oleracea ) Juice as an Anticonvulsant Agent : In Vitro Mechanistic Study of GABAergic Targets[J]. Oxidative medicine and, cellular longevity, 2018, 2018(3):1-6.

[13] Masaru Nagaoka. Fano compactifications of contractible affine 3-folds with trivial log canonical divisors [J]. International Journal of Mathematics, 2018, 29: 28-61.

[14] Vichi Stefania, Pizzale Lorena, Toffano Emilio. Detection of Hazelnut Oil in Virgin Olive Oil by Assessment of Free Sterols and Triacylglycerols[J]. Journal of Aoac International, 2019(5):5.

[15] E Fanoy, F Dijkstra, W van der Hoek. Familiarity of general practitioners with Q fever decreases hospitalisation risk [J]. Netherlands Journal of Medicine, 2018, 76(4):184-189.

[16] Shi, Chenyuyi He, Xiaoyong Peng, Jun Xiao, Guina Liu, Feng Lin, Fangting Zhang, Hao. Tunable terahertz hybrid graphene-metal patterns metamaterials [J]. Optics \& Laser Technology, 2019, 114:28-34.

[17] Lucas Chesnel, Sergei A. Nazarov. Non reflection and perfect reflection via Fano resonance in waveguides [J]. Communications in mathematical sciences, 2018, 16(7).

[18] Tun Cao, Yimei Qiu. Lateral sorting of chiral nanoparticles using Fano-enhanced chiral force in visible region [J]. Nanoscale, 2018, 10.

[19] He, J. , Fan, C. , Wang, J. , Ding, P. , Cai, G. , \& Cheng, Y. A giant localized field enhancement and high sensitivity in an asymmetric ring by exhibiting Fano resonance [J]. Journal of Optics, 2013, 15(2):025007. 
[20] Zicong, G., Kunhua, W. , Qinyang, H. , Wenhui, L. , Jiyan, L. , \& Yihong, F. . Plasmonic Multichannel Refractive Index Sensor Based on Subwavelength Tangent-Ring Metal-Insulator-Metal Waveguide[J]. Sensors, 2018, 18(5):1348-1357.

[21] Zhang Zhaojian, Yang Junbo, He Xin, Zhang Jingjing, Huang Jie, Chen Dingbo, Han Yunxin. Plasmonic Refractive Index Sensor with High Figure of Merit Based on Concentric-Rings Resonator[J]. Sensors, 2018, 18(2):116-125.

[22] Wang, Q. , Ouyang, Z. , Lin, M. , \& Liu, Q. . Independently Tunable Fano Resonances Based on the Coupled Hetero-Cavities in a Plasmonic MIM System[J]. Materials, 2018, 11(9).

[23] Kim, K. Y. , Cho, Y. K. , Tae, H. S. , \& Lee, J. H. . Light transmission along dispersive plasmonic gap and its subwavelength guidance characteristics [J]. Optics Express, 2006, 14(1):320-30.

[24] Chen, L. , Liu, Y. , Yu, Z. , Wu, D. , Ma, R. , \& Zhang, Y. Numerical analysis of a near-infrared plasmonic refractive index sensor with high figure of merit based on a fillet cavity[J]. Optics Express, 2016, 24(9):9975.

[25] Zhidong, Zhang, Liang, Luo, Chenyang, Xue, Wendong, Shubin, Yan. Fano Resonance Based on Metal-Insulator-Metal Waveguide-Coupled Double Rectangular Cavities for Plasmonic Nanosensors[J]. Sensors, 2016, 16(5):642-652.

[26] Shu-Bin, Y. , Liang, L. , Chen-Yang, X. , \& Zhi-Dong, Z. . A Refractive Index Sensor Based on a Metal-Insulator-Metal Waveguide-Coupled Ring Resonator[J]. Sensors, 15(11):29183-29191.

[27] Yongjun Liu, Daqing Zhu, ZhenYu Yang, Na Ning, Zhene Xu, Dongsheng Lu, Aiqing Zhang. Relationship between the effective refractive index and pore ratio of nano-porous polymer film[C]// Nano-Optics and Nano-Structures. International Society for Optics and Photonics, 2002.

[28] Li, L. , Liu, G. Q. , Huang, K. , Chen, Y. H. , Gong, L. X. , \& Tang, F. L. The water content sensor in heavy oil based on one-dimensional photonic crystals [J]. Optik - International Journal for Light and Electron Optics, 2013, 124(16):2519-2521.

[29] Tittl A, Giessen H , Liu N . Plasmonic gas and chemical sensing[J]. Nanophotonics, 2014, 3(3):157-180. 
Figures

(a)

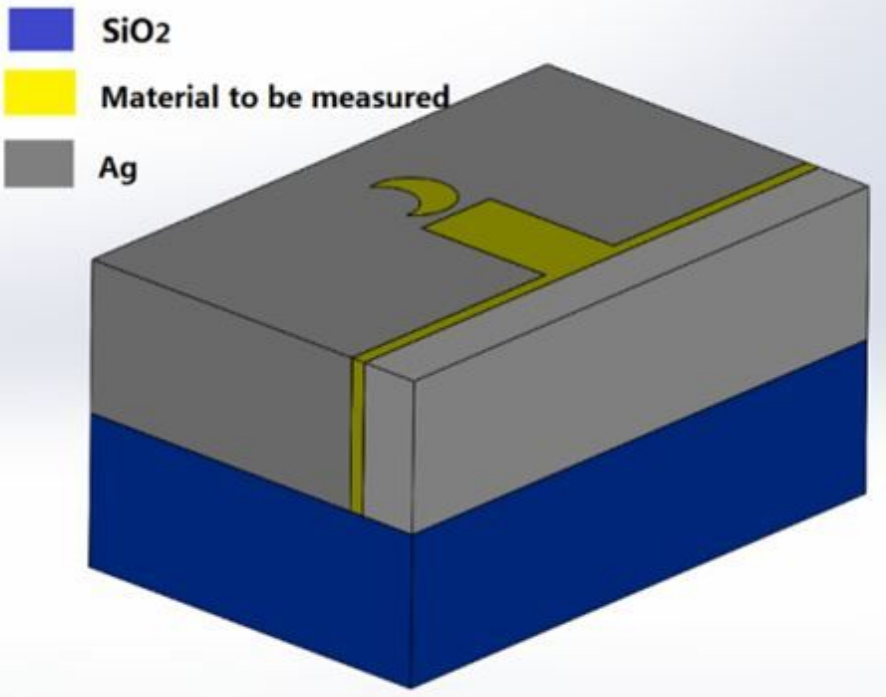

(b)

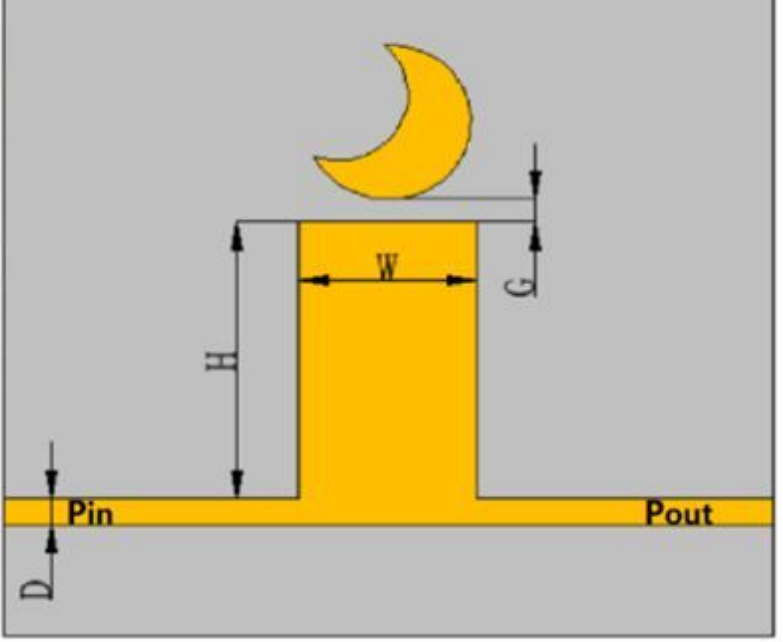

Figure 1

$3 \mathrm{D}$ and $2 \mathrm{D}$ renderings of the system

(a)

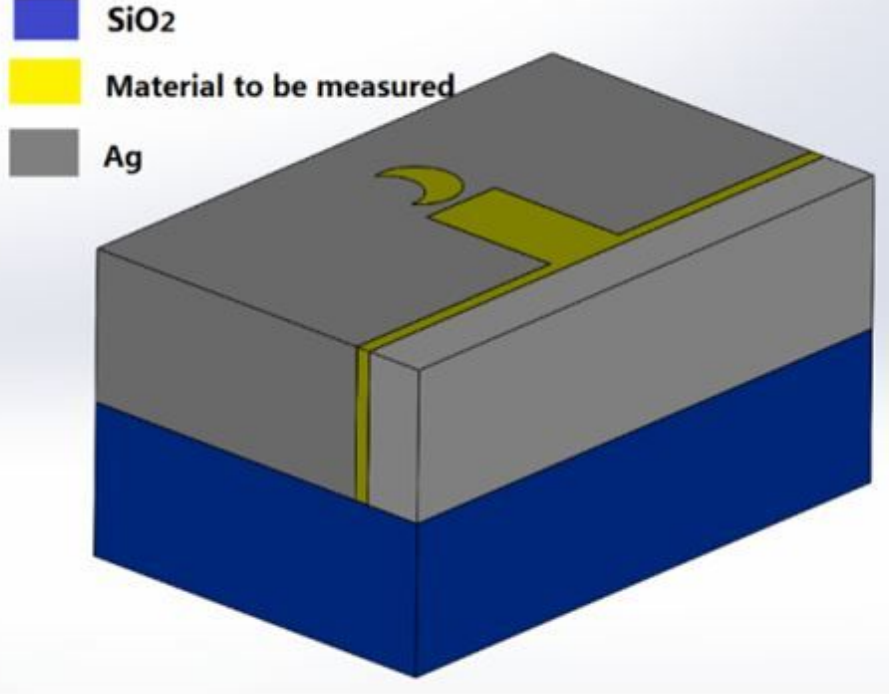

(b)

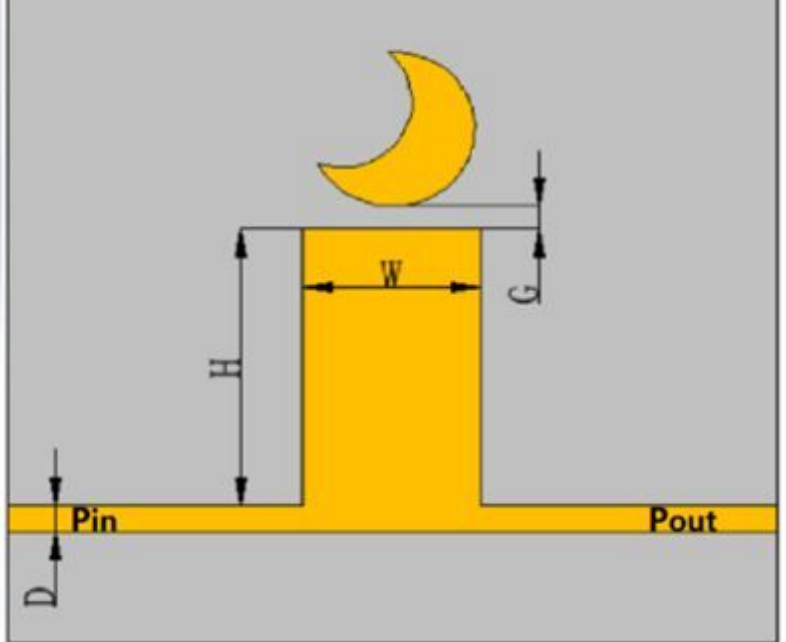

Figure 1

$3 \mathrm{D}$ and $2 \mathrm{D}$ renderings of the system 


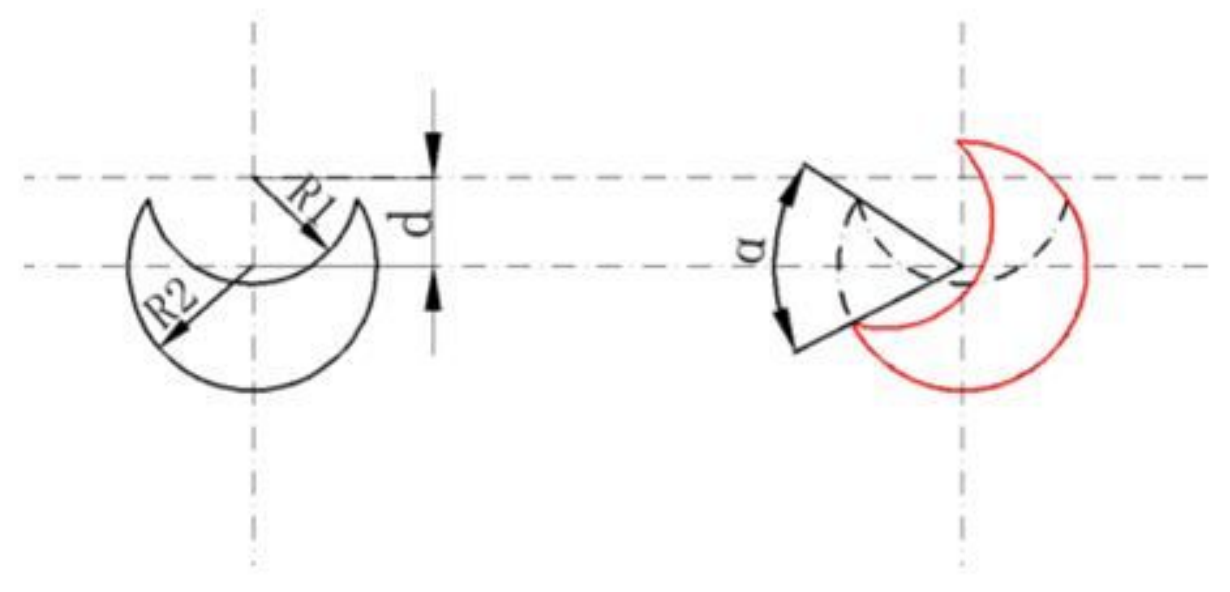

Figure 2

Schematic diagram showing the structure of the crescent-shaped cavity resonator.

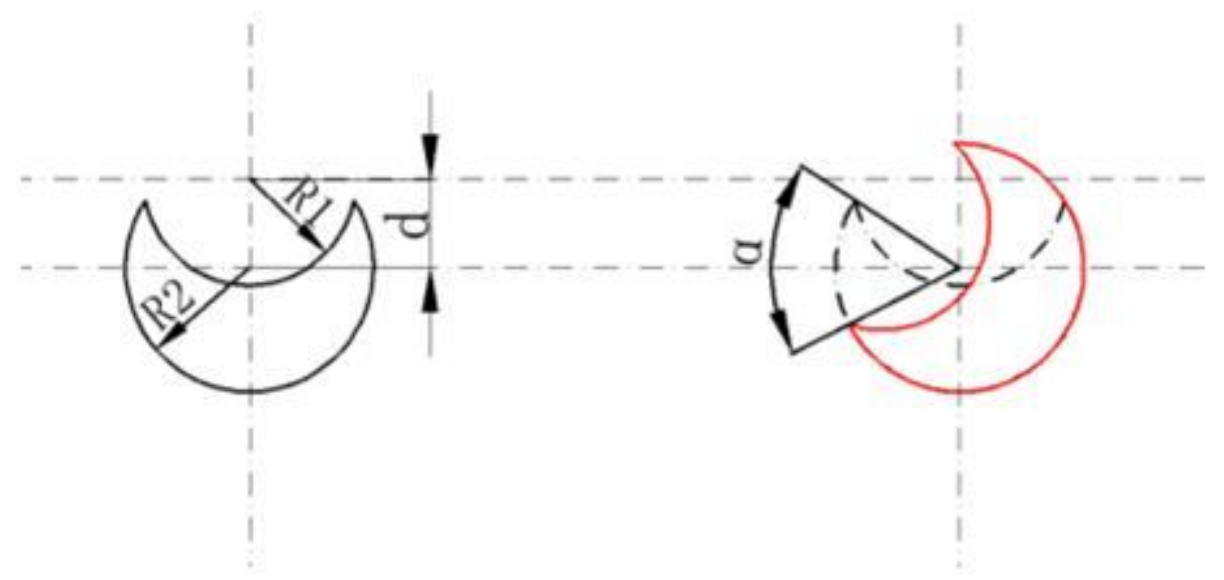

Figure 2

Schematic diagram showing the structure of the crescent-shaped cavity resonator. 

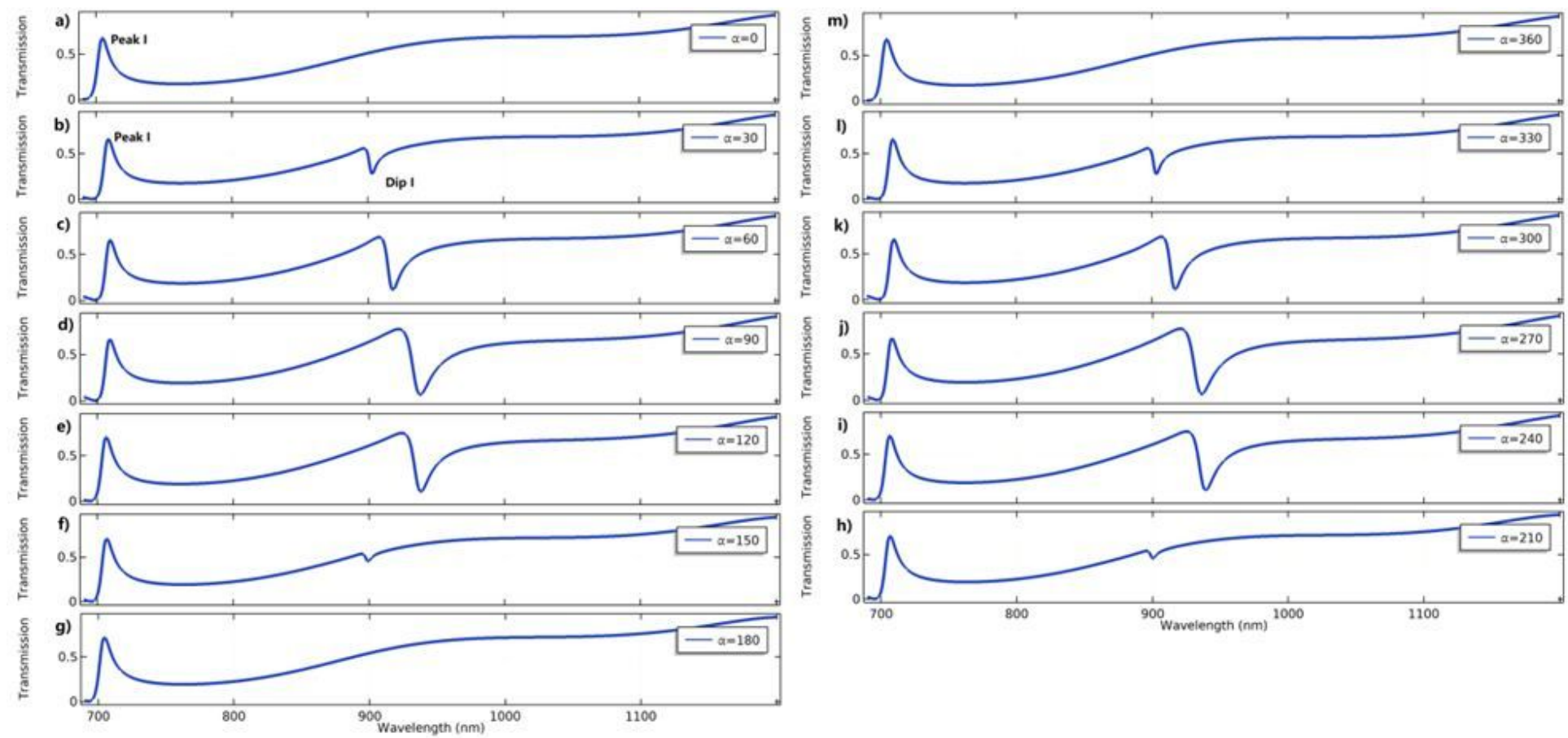

\section{Figure 3}

Transmission spectra from the designed structure at different the rotation angle a. Figure A-C is the magnetic field distribution diagram
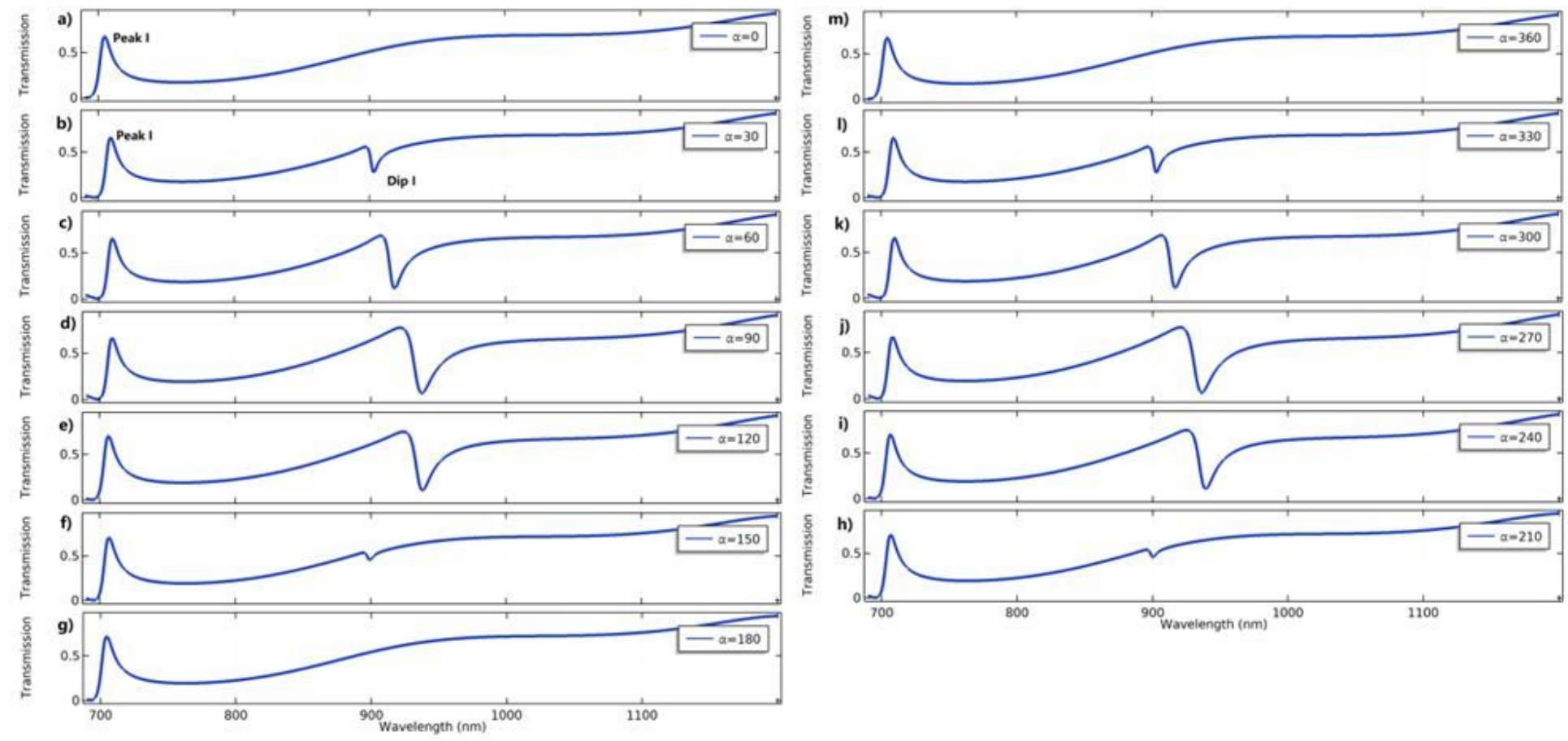

Figure 3

Transmission spectra from the designed structure at different the rotation angle a. Figure A-C is the magnetic field distribution diagram 

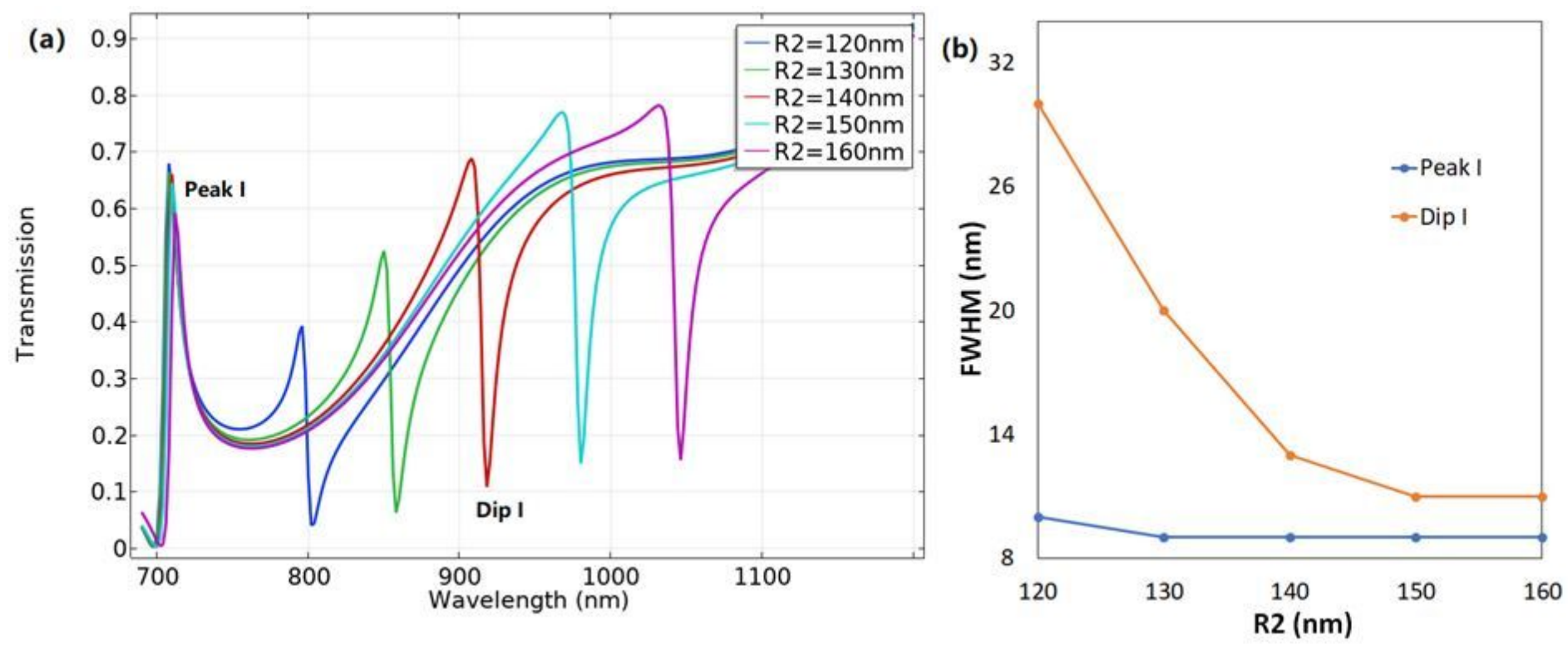

\section{Figure 4}

(a) The transmission spectra of R2 $\otimes$ (b) FWHM with the Fano resonance lines.
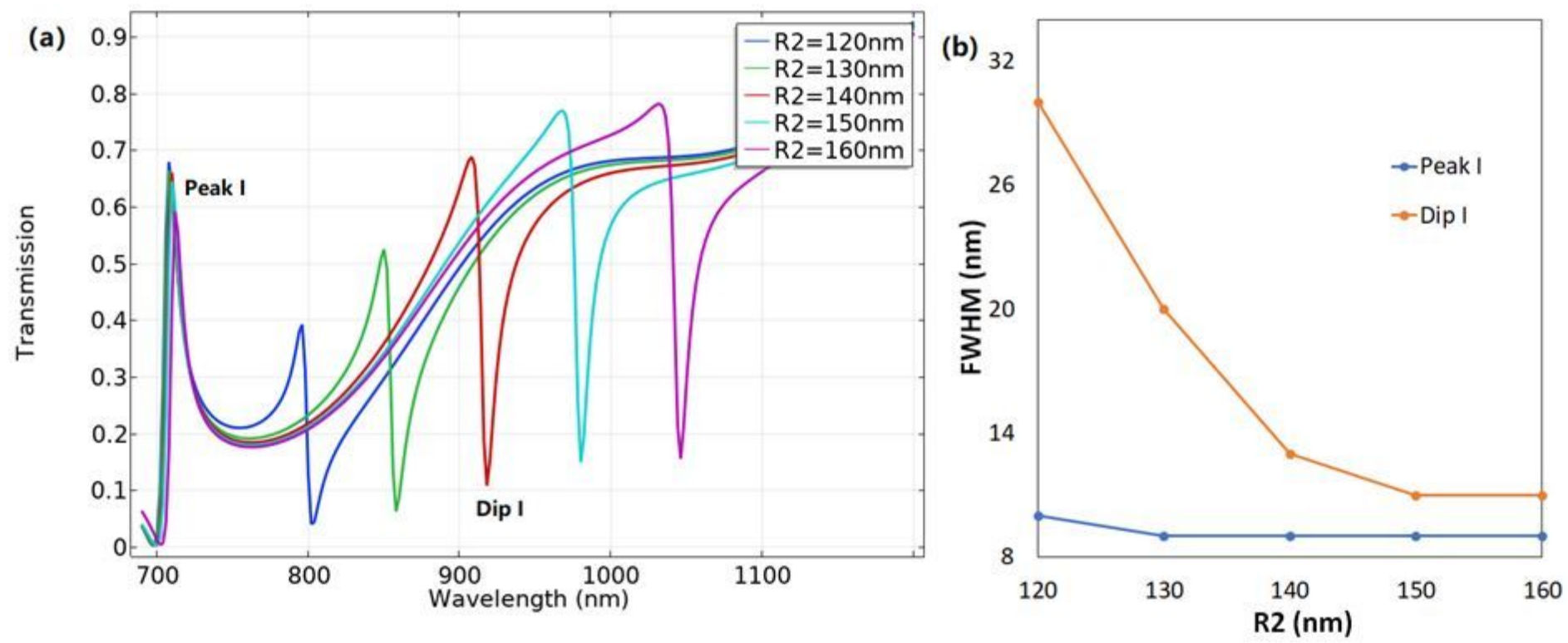

Figure 4

(a) The transmission spectra of R2 $\otimes$ (b) FWHM with the Fano resonance lines. 

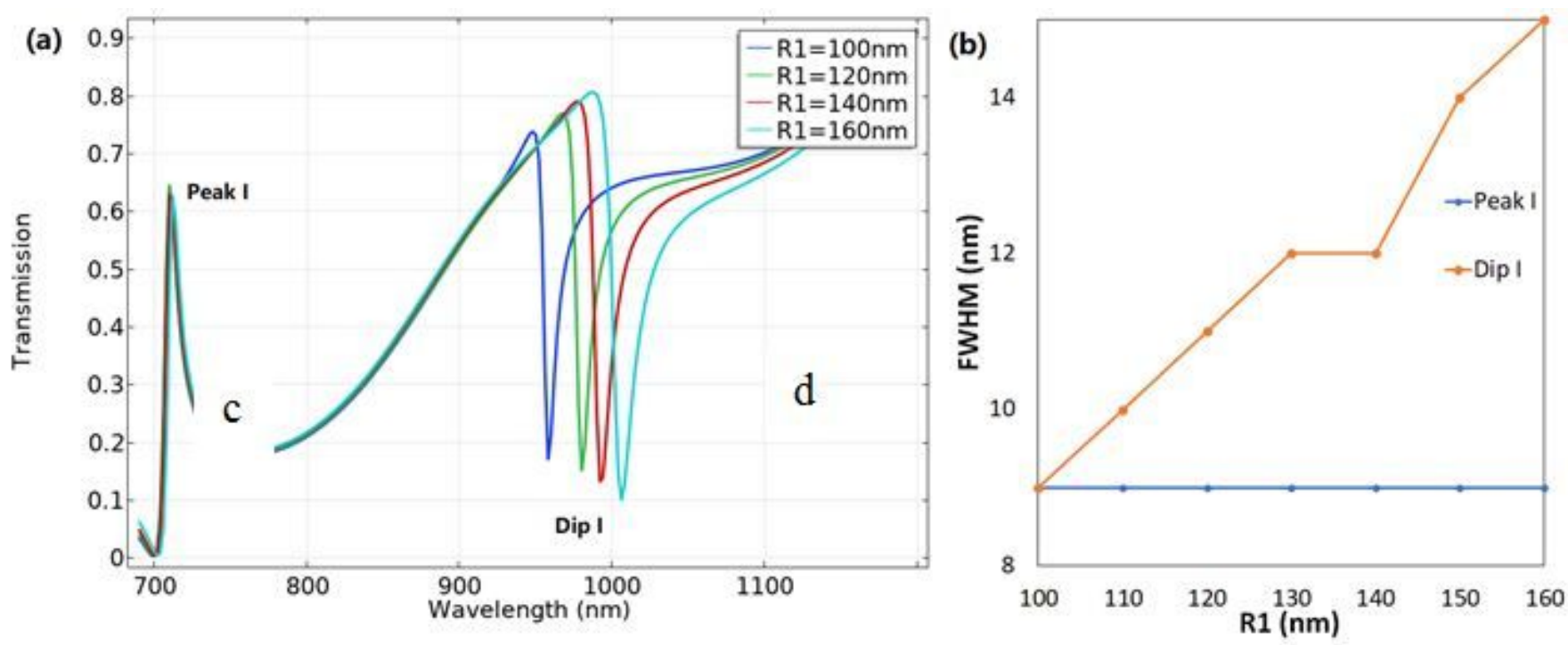

(c)
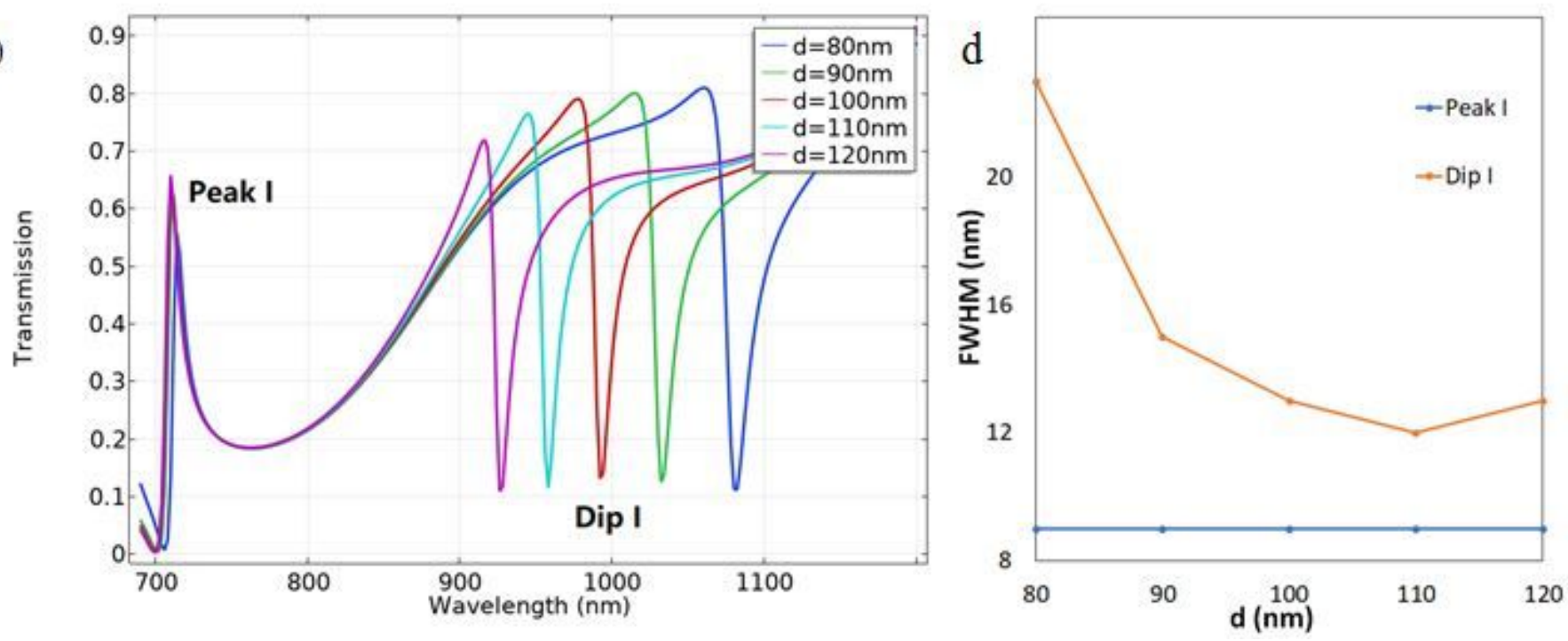

\section{Figure 5}

(a) The transmission spectra of R1 (b) R1 and FWHM. (c) Transmission spectra of d. (d) d and FWHM 

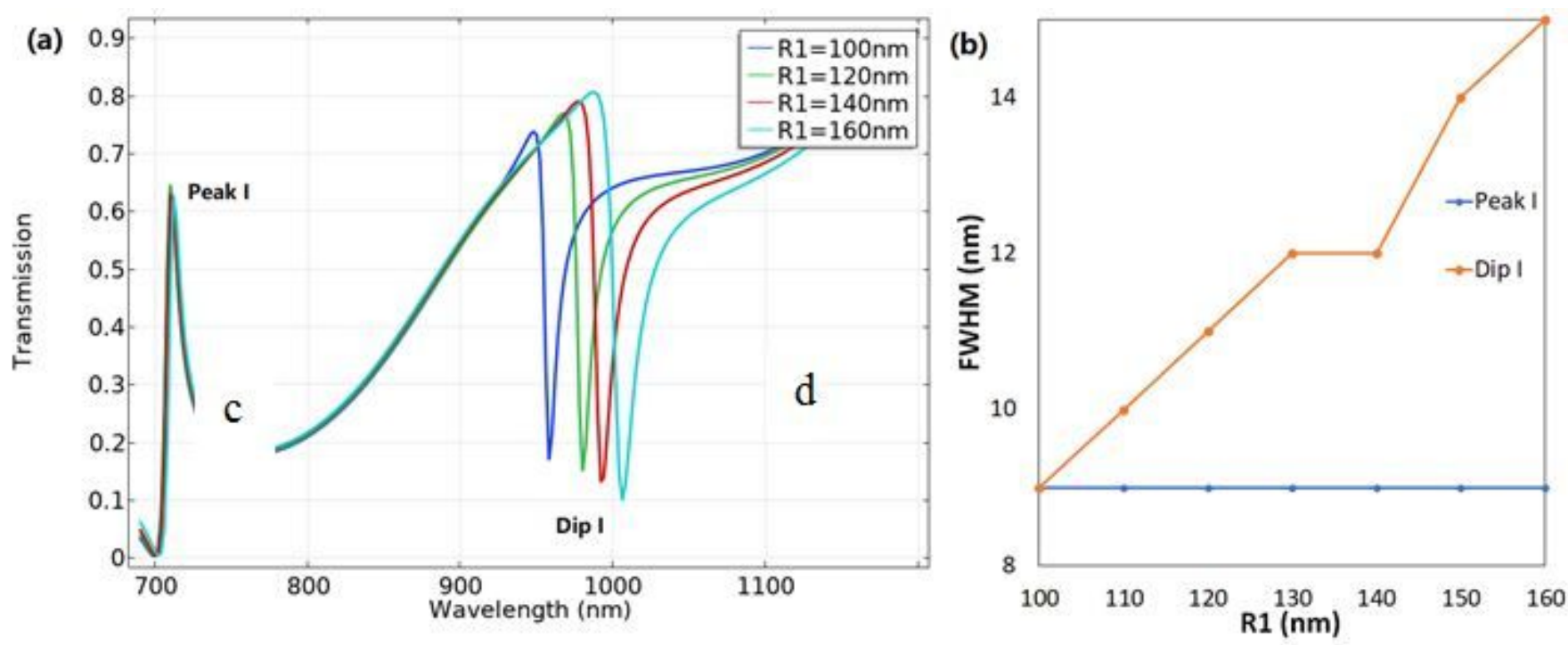

(c)
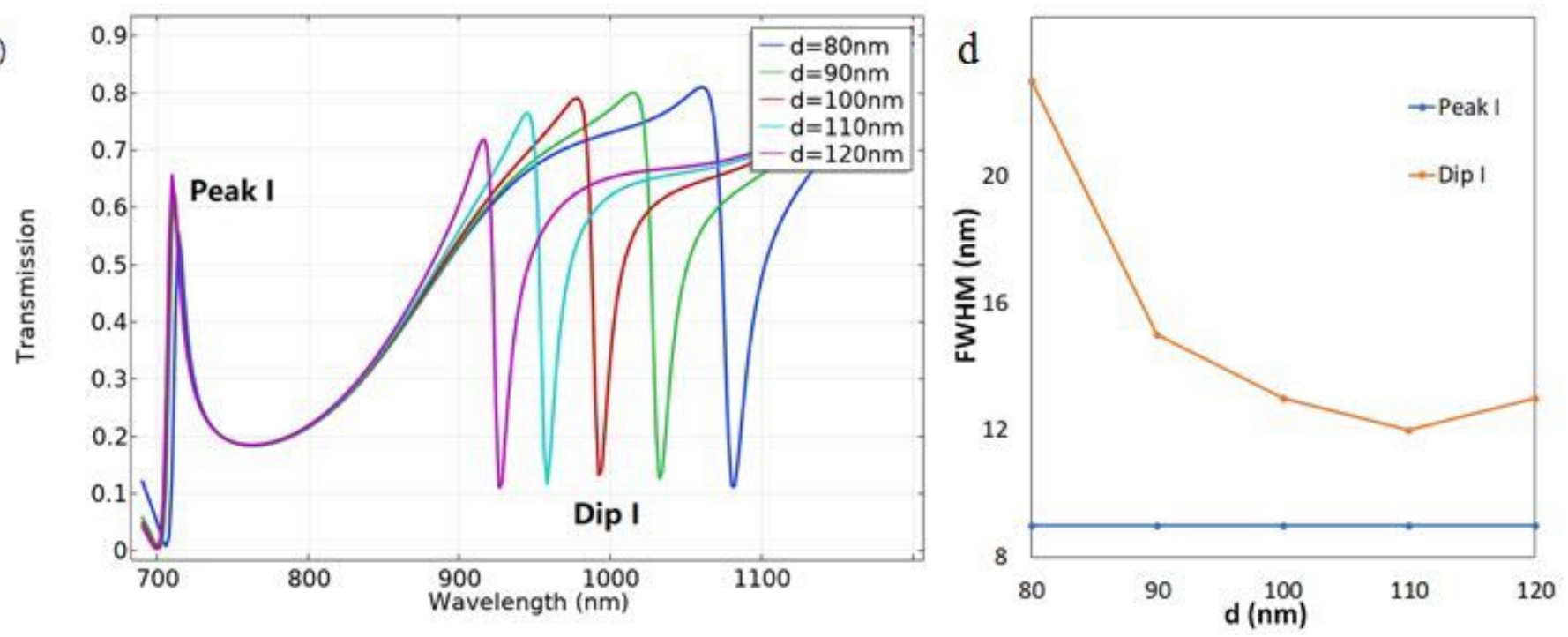

\section{Figure 5}

(a) The transmission spectra of R1 (b) R1 and FWHM. (c) Transmission spectra of d. (d) d and FWHM
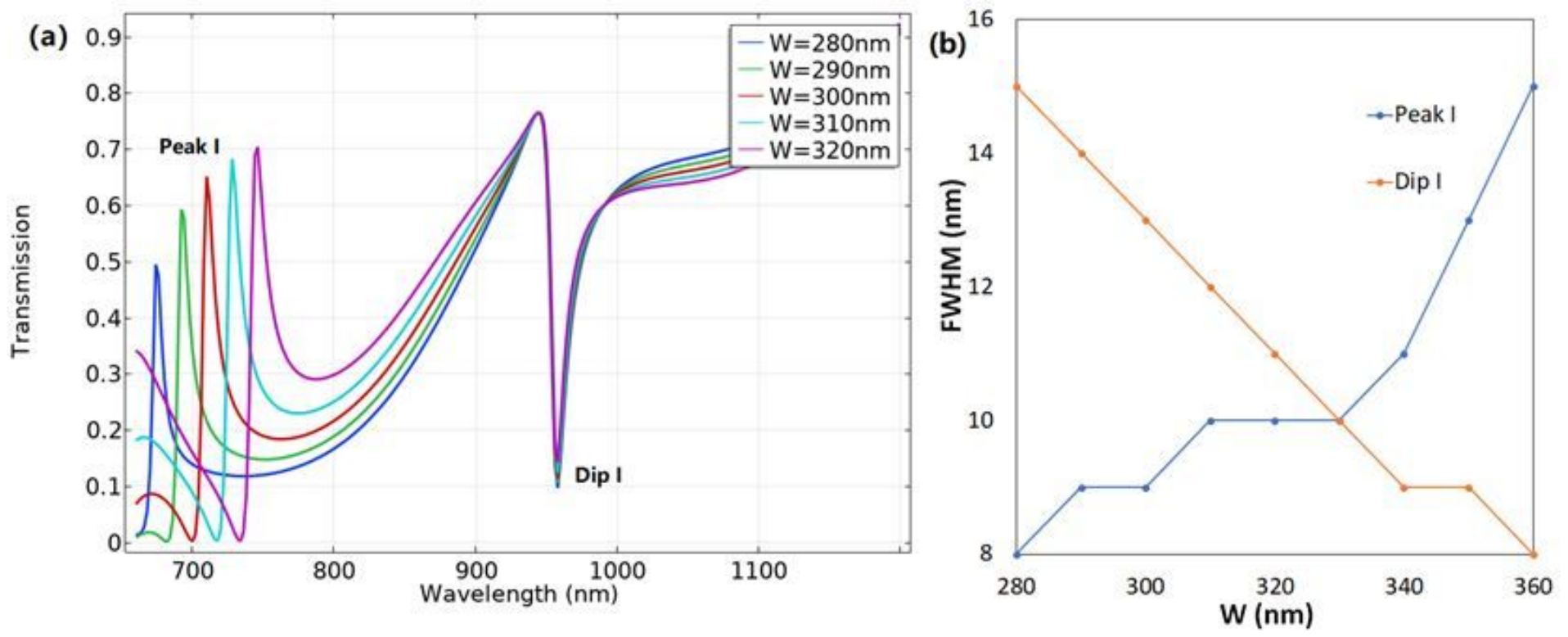
Figure 6

(a) Transmission spectra a of the rectangular cavity resonator (b)W and FWHM
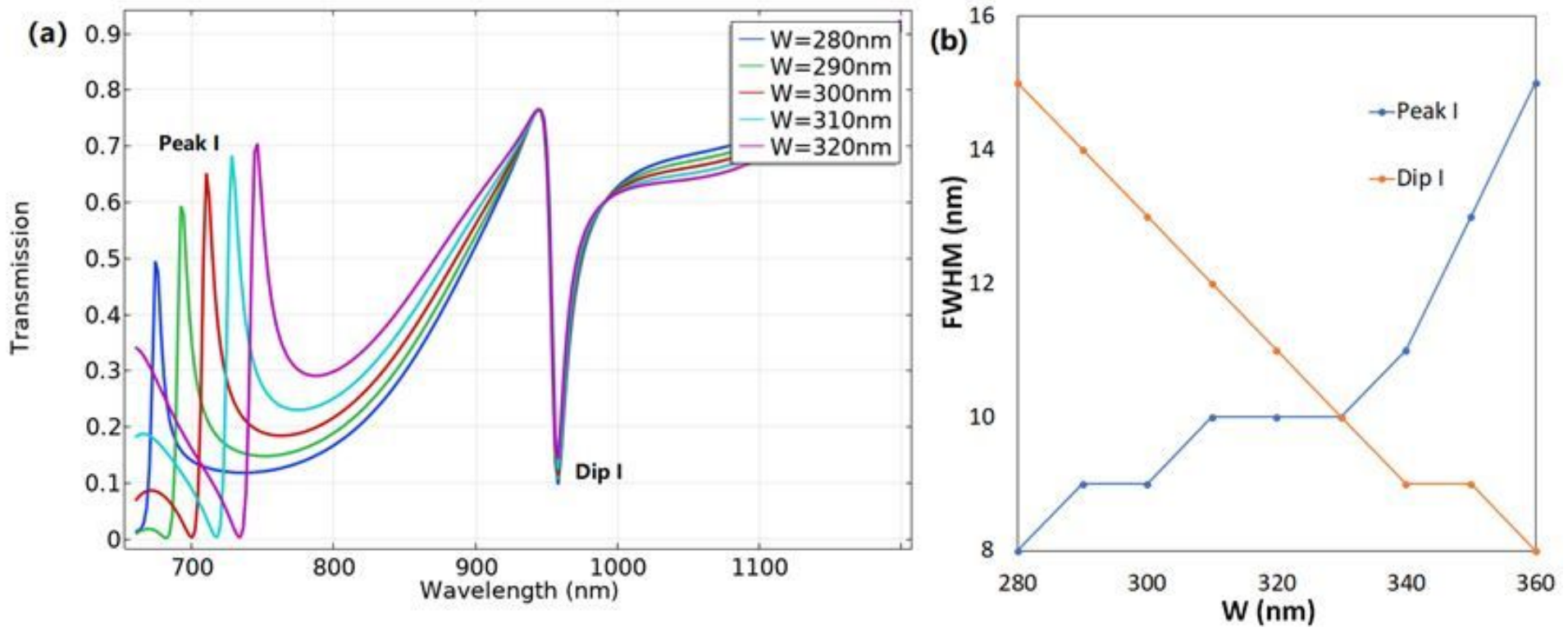

Figure 6

(a) Transmission spectra a of the rectangular cavity resonator (b)W and FWHM
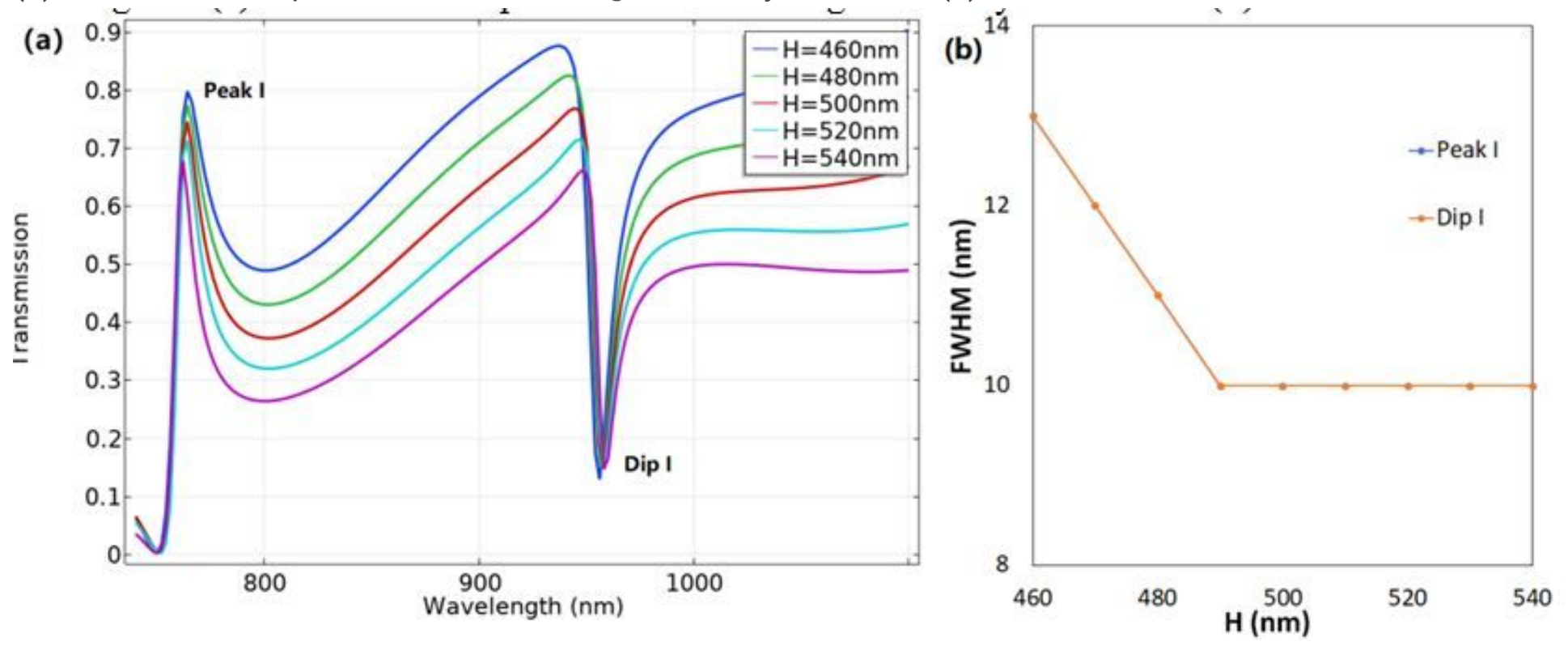

Figure 7

(a) Transmission spectra of the height $\mathrm{H}$ (b) $\mathrm{H}$ and FWHM 

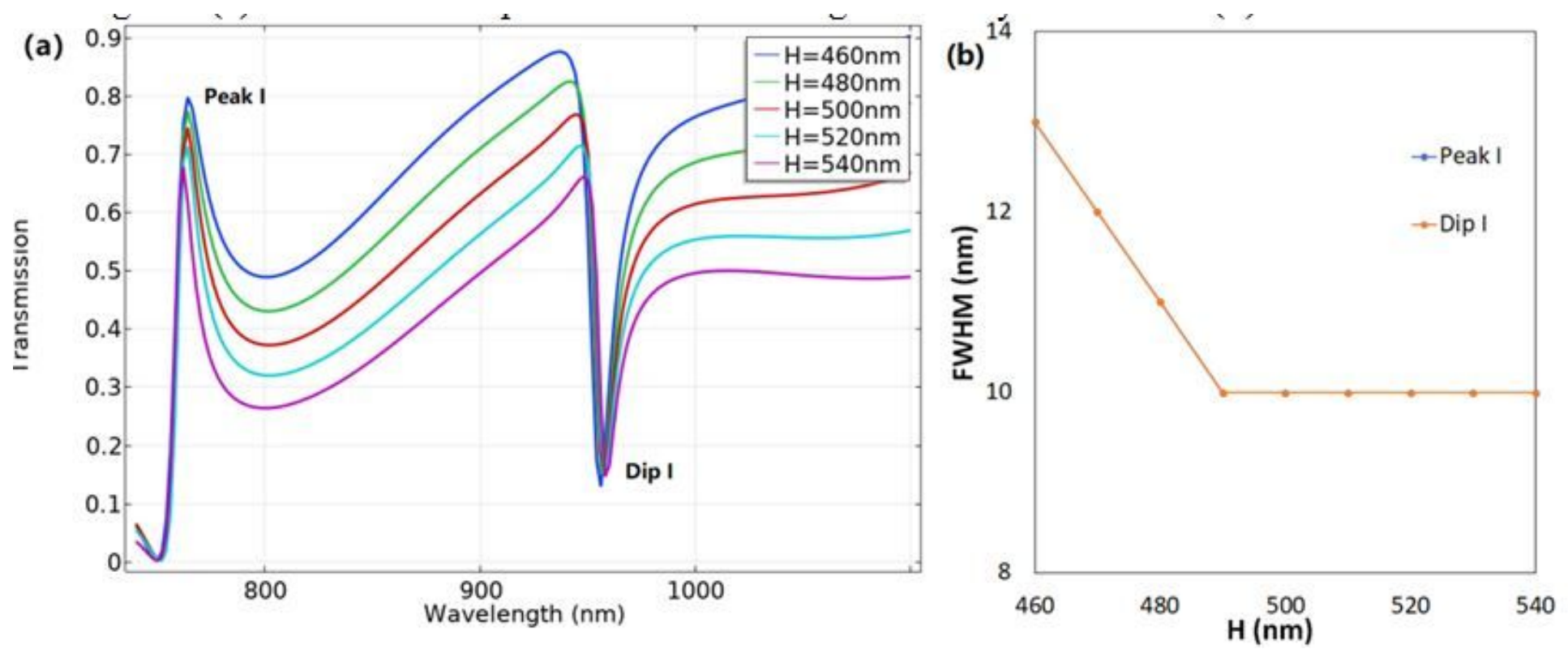

Figure 7

(a) Transmission spectra of the height $\mathrm{H}$ (b) $\mathrm{H}$ and FWHM
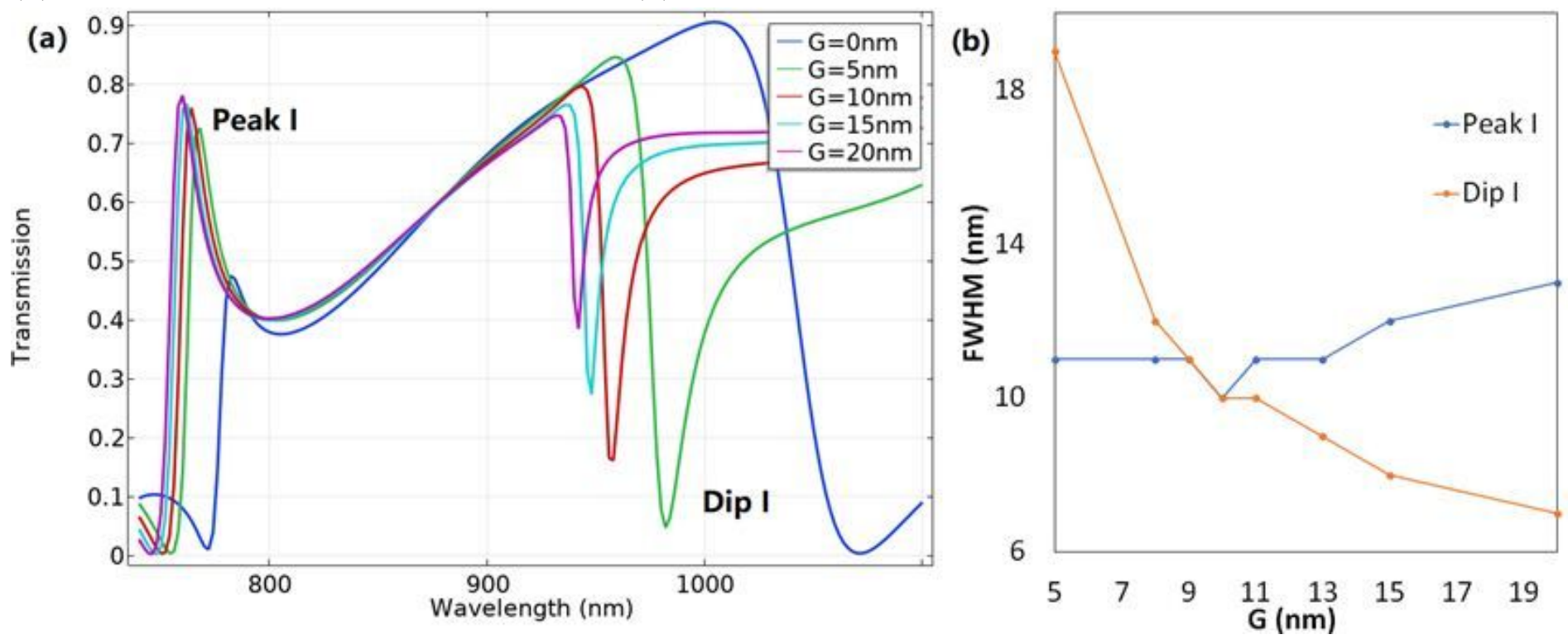

Figure 8

(a) Transmission spectra with distance G (b) G and FWHM 

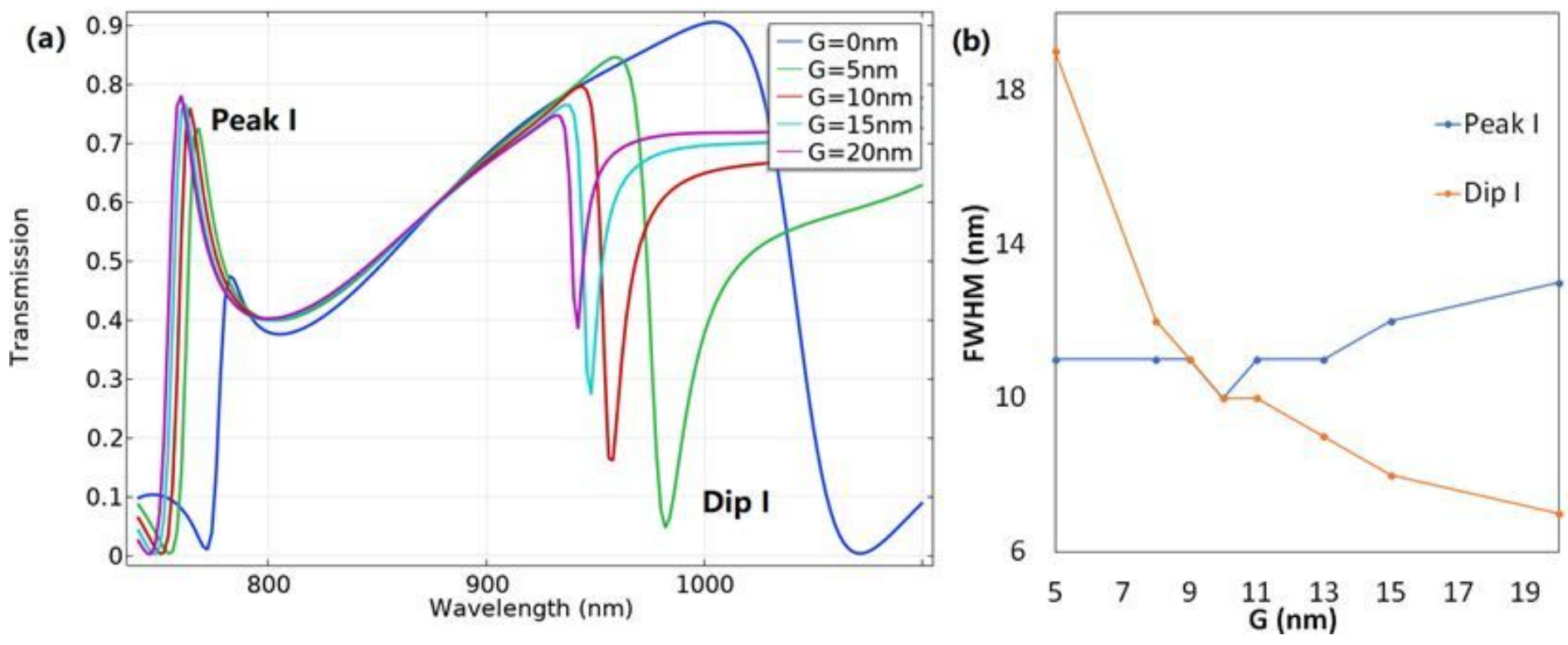

Figure 8

(a) Transmission spectra with distance G (b) G and FWHM
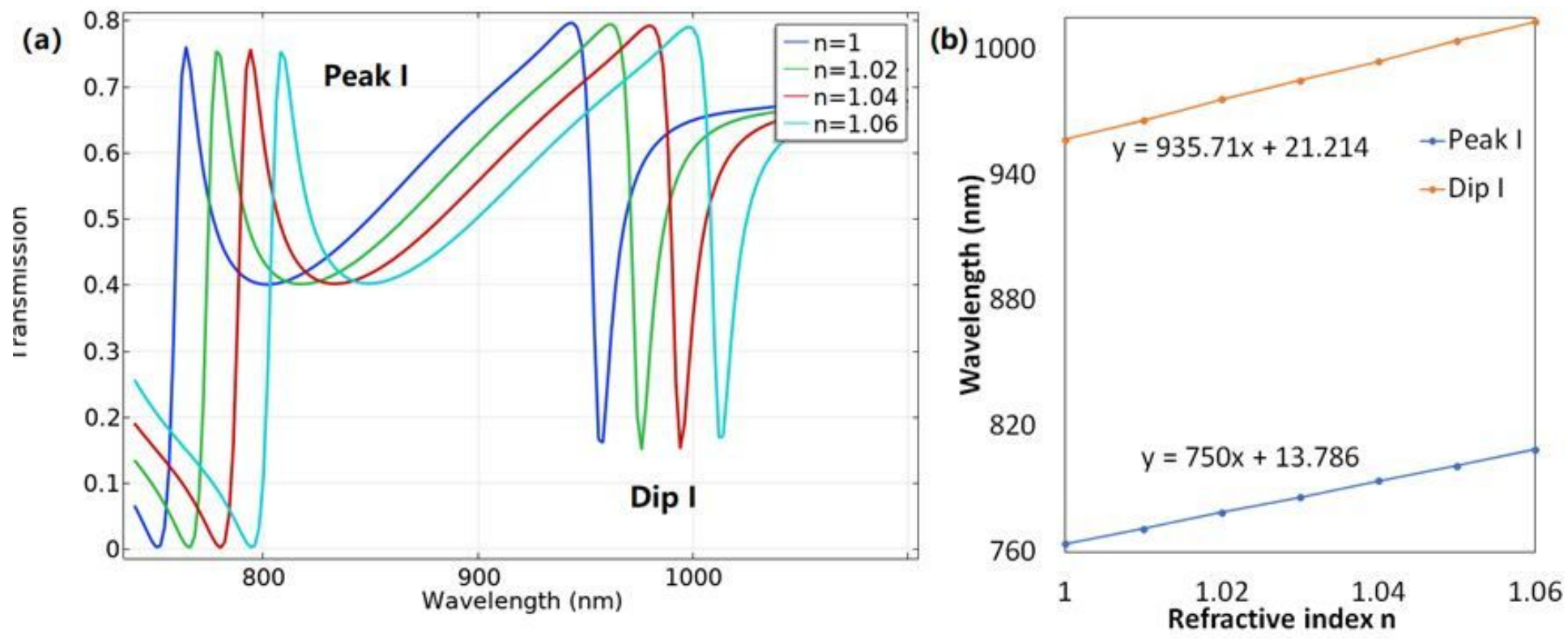

Figure 9

(a) Transmission spectra as the refractive index n. (b) Fano resonance wavelength with refractive index 

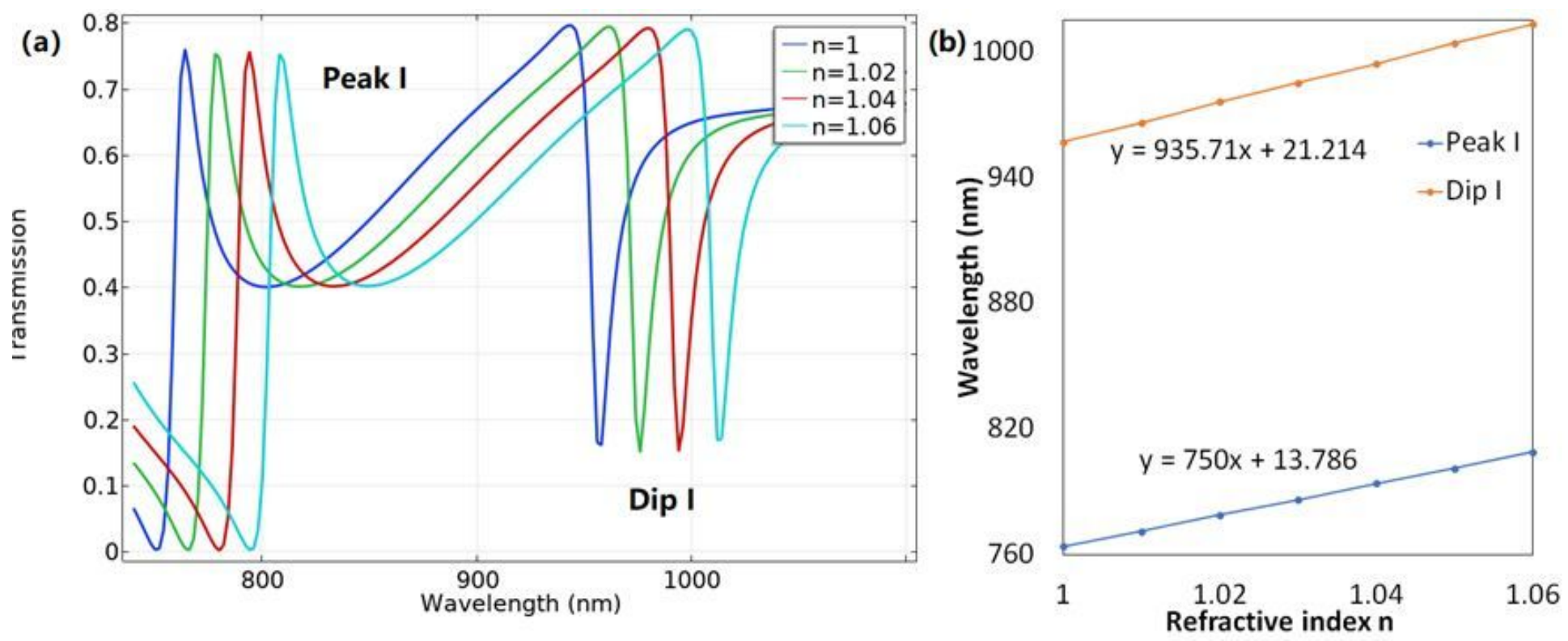

Figure 9

(a) Transmission spectra as the refractive index $n$. (b) Fano resonance wavelength with refractive index

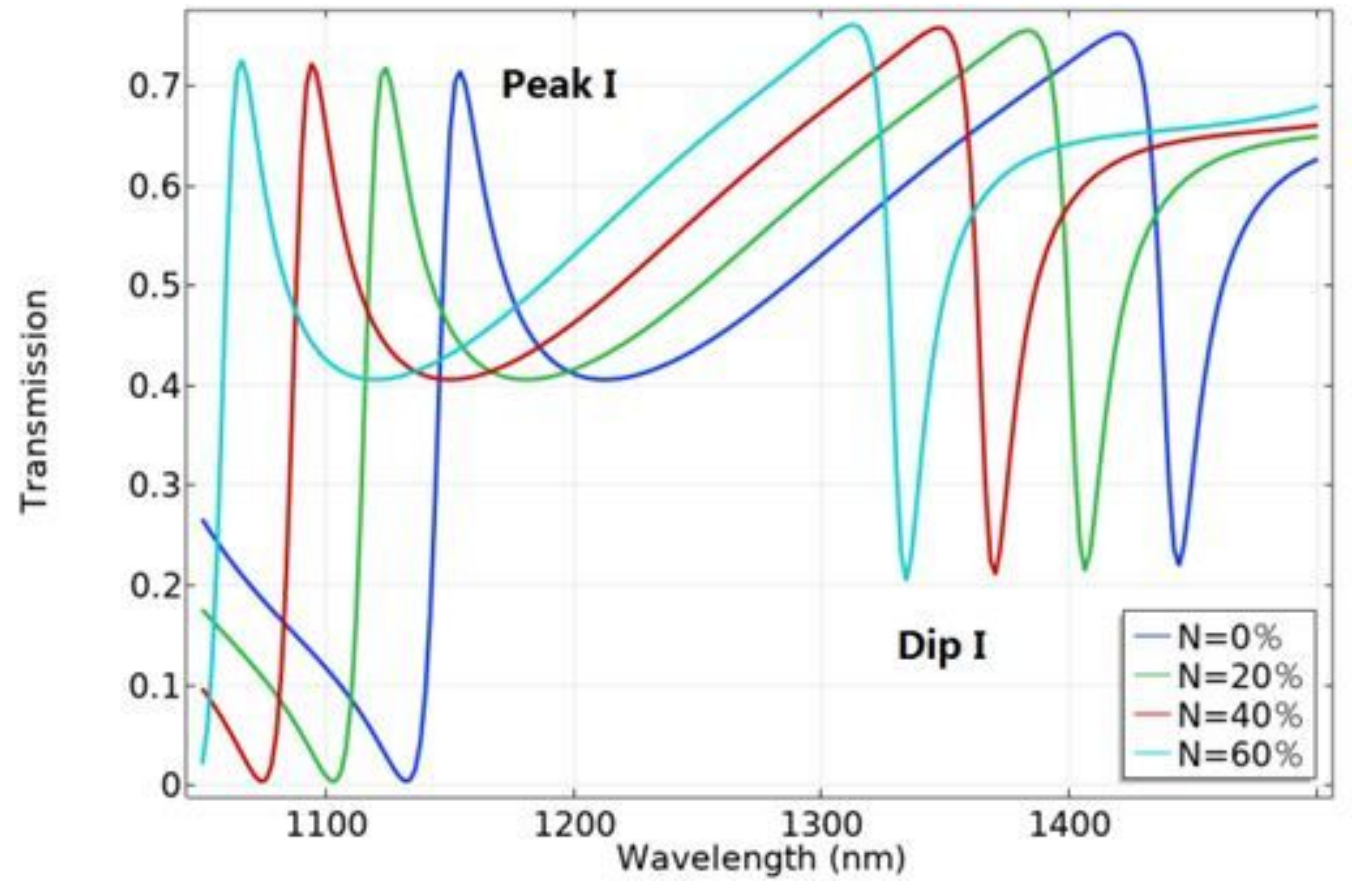

Figure 10

Heavy oils with different water content 


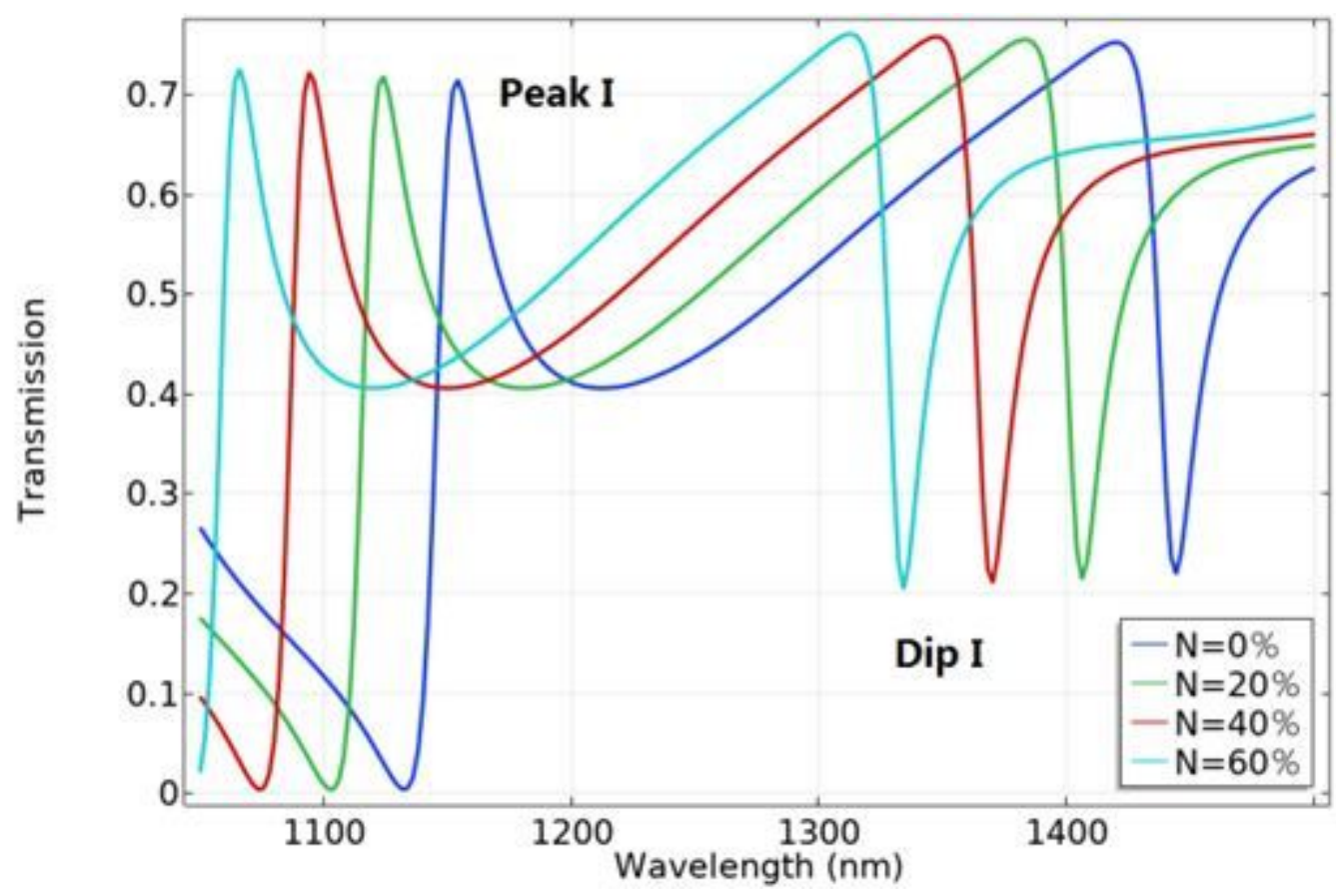

Figure 10

Heavy oils with different water content

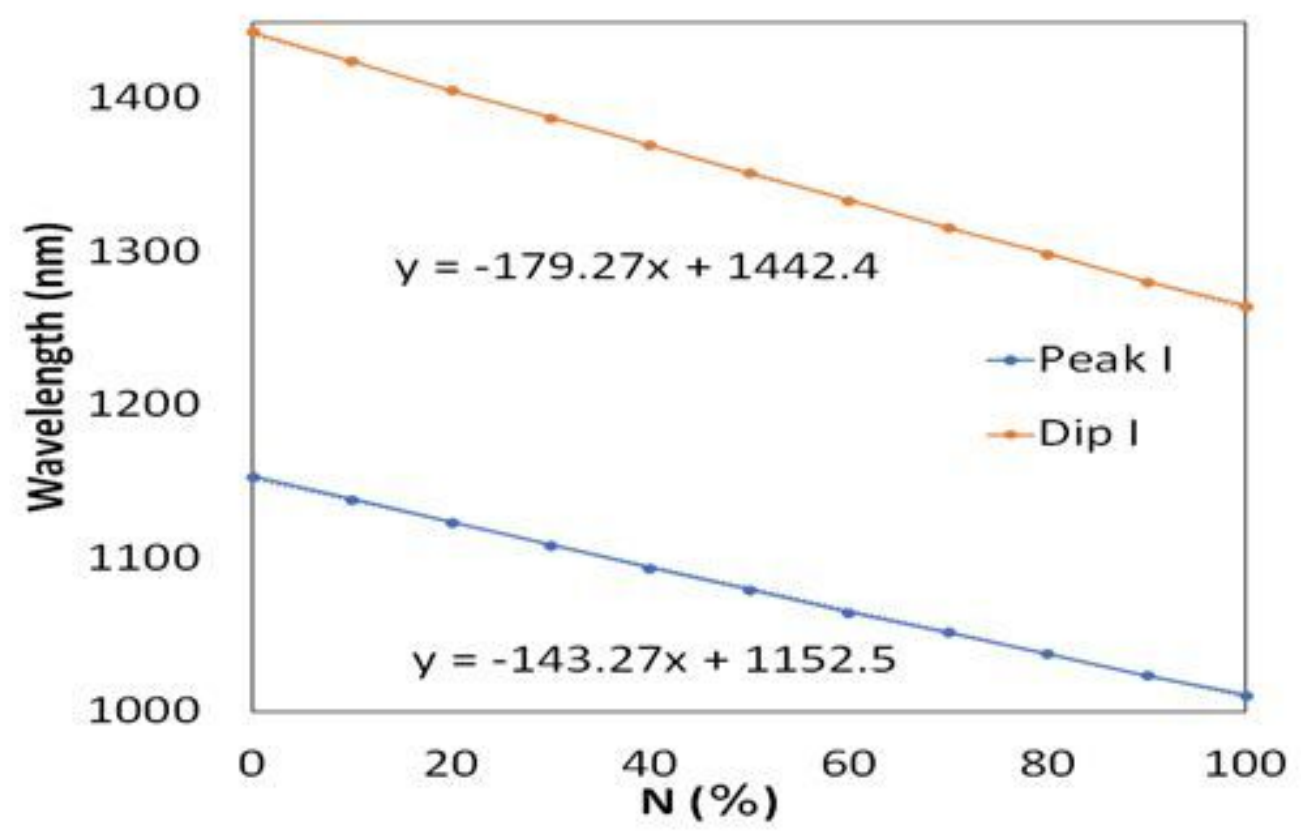

Figure 11

Volume fraction of water with the Fano resonance wavelength. 


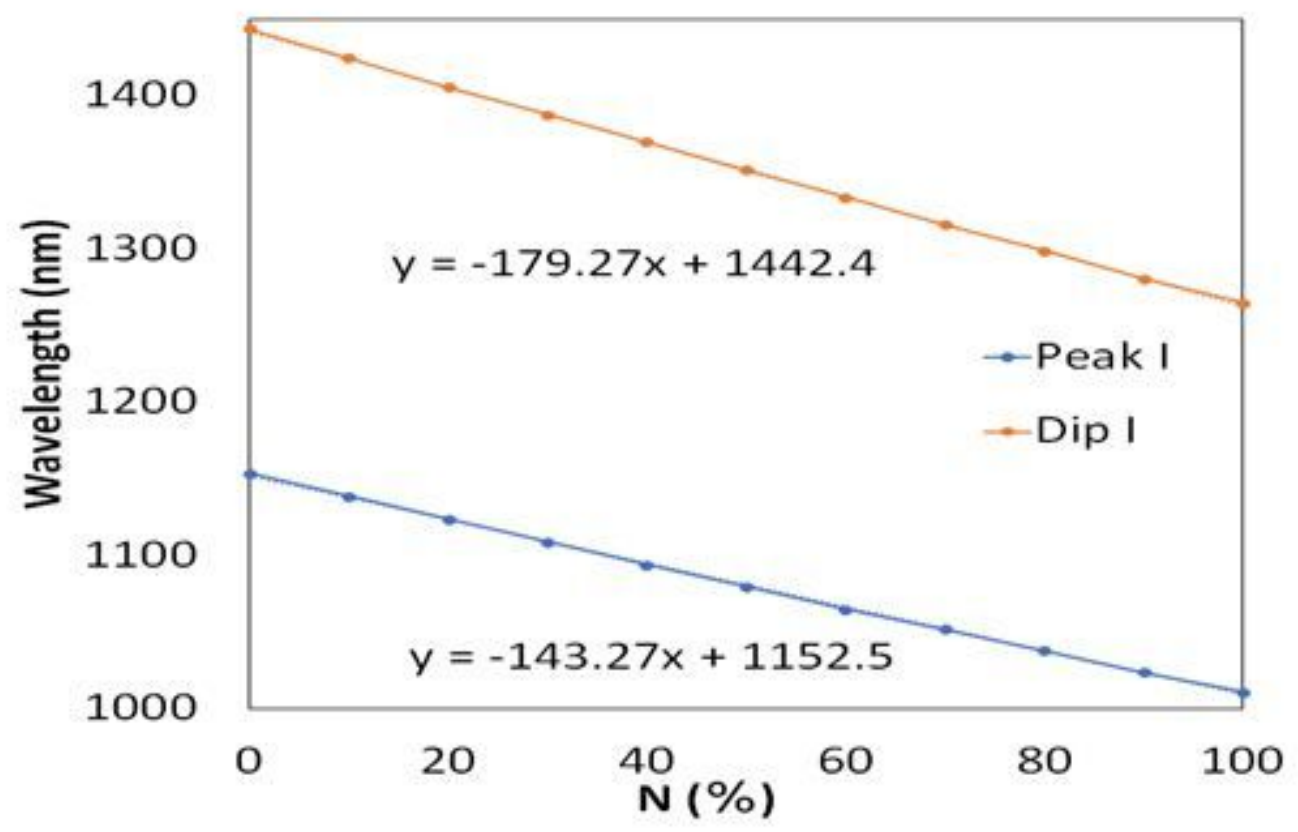

Figure 11

Volume fraction of water with the Fano resonance wavelength. 